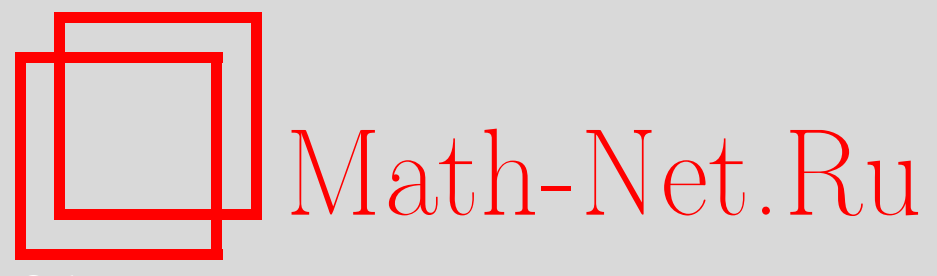

В. Н. Безверхний, И. В. Добрынина, Решение проблемы обобщенной сопряженности слов в группах Кокстера большого типа, Дискрет. матем., 2005, том 17, выпуск $3,123-145$

DOI: https://doi.org/10.4213/dm122

Использование Общероссийского математического портала Math-Net.Ru подразумевает, что вы прочитали и согласны с пользовательским соглашением http://www . mathnet.ru/rus/agreement

Параметры загрузки:

IP: 54.205 .225 .156

26 апреля 2023 г., 09:20:55 


\title{
Решение проблемы обобщенной сопряженности слов в группах Кокстера большого типа
}

\author{
() 2005 г. В. Н. Безверхний, И. В. Добрынина
}

Для групп Кокстера большого типа решена проблема обобщенной сопряженности слов, доказано, что централизатор конечно порожденной подгруппы конечно порожден и существует алгоритм, выписывающий его образующие. Показано, что в общем случае централизатор конечно порожденной группы не является циклическим. Построен пример группы данного класса, не являющейся гиперболической группой.

Работа выполнена при поддержке Министерства образования России, грант Рд 02-1.1-209.

Группа $G$, заданная системой образующих $a_{i}, i \in J,|J|<\infty$, и системой определяющих соотношений $a_{i}^{2}=1$ для всех $i \in J,\left(a_{i} a_{j}\right)^{m_{i j}}=1, i \neq j, i, j \in J, m_{i j}$ - элемент матрицы Кокстера $\left(m_{i j}\right), i, j \in J$, соответствующей данной группе (см. [1]), причем $m_{i j} \geqslant 3$ для $i \neq j$, называется группой Кокстера большого типа. В случае $m_{i j}>3$ имеем группу Кокстера экстра большого типа.

Теорема 1 ([1]). В группах Кокстера экстра большого типа разрешима проблема сопряженности слов.

Нашей целью является решение проблемы обобщенной сопряженности слов и описание централизатора конечно порожденной подгруппы в группах Кокстера большого типа. Проблема сопряженности слов для данного класса групп решена авторами в [2].

Будем говорить, что в группе $G$ разрешима проблема обобщенной сопряженности слов, если существует алгоритм, позволяющий установить, существует ли такое $z \in G$, что $\boldsymbol{\alpha}_{i=1}^{n}\left(z^{-1} w_{i} z=v_{i}\right)$ для любых двух конечных множеств слов $\left\{w_{i}\right\}_{i=1, \ldots, n},\left\{v_{i}\right\}_{i=1, \ldots, n}$ из $G$.

Пусть $F_{i}=\left\langle a_{i} ; a_{i}^{2}\right\rangle, F=*_{i=1}^{n} F_{i}$ - свободное произведение циклических групп порядка 2. Отождествим каждый образующий $a_{i}$ группы $F$ с его обратным $a_{i}^{-1}$. Слово $w=a_{i_{1}} \ldots a_{i_{n}}$ группы $F$ называется приведенным, если индексы рядом стоящих букв $a_{i_{j}}$ и $a_{i_{j+1}}$ записи $w$ различны; длина $w$ равна $n$.

Пусть $m_{i j}<\infty$ и $r_{i j}=\left(a_{i} a_{j}\right)^{m_{i j}}$, тогда в $F$ существуют в точности две различные перестановки $r_{i j}: r_{i j}=\left(a_{i} a_{j}\right)^{m_{i j}}$ и $r_{j i}=\left(a_{j} a_{i}\right)^{m_{i j}}, i \neq j$.

Обозначим через $F_{i j}$ группу $F_{i j}=F_{i} * F_{j}$, через $G_{i j}$ группу Кокстера большого типа $G_{i j}=\left\langle a_{i}, a_{j} ; a_{i}^{2}, a_{j}^{2}, r_{i j}, r_{j i}\right\rangle$.

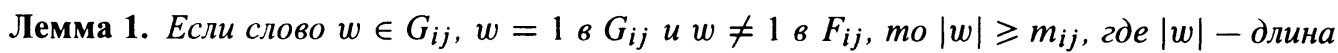
слова $w$. 
Доказательство очевидно.

Лемма 2. Пусть $w \in G_{i j}, w=w_{1} w_{2}, w=1$ в $G_{i j} u w \neq 1$ в $F_{i j}$. Тогда

(a) если $\left|w_{1}\right| \leqslant m_{i j}$, то $\left|w_{1}\right| \leqslant\left|w_{2}\right|$;

(б) если $\left|w_{1}\right|<m_{i j}$, по $\left|w_{1}\right|<\left|w_{2}\right|$.

Справедливость леммы 2 следует из леммы 1.

Обозначим через $R_{i j}$ множество всех нетривиальных слов, циклически приведенных в свободном произведении $F_{i j}$ и равных 1 в группе $G_{i j}$. Элемент $r \in R_{i j}$ будем называть соотношением типа $(i, j)$.

Лемма 3. Любое слово, принадлежащее $R_{i j}$, является степенью либо $r_{i j}$, либо $r_{j i}$.

Доказательство. Положим

$$
r_{i j}^{\varepsilon}= \begin{cases}r_{i j}, & \text { если } \varepsilon=1, \\ r_{j i}, & \text { если } \varepsilon=-1\end{cases}
$$

Пусть $w \in R_{i j}$. Тогда

$$
w=\left(s_{1} r_{i j}^{\varepsilon_{1}} s_{1}^{-1}\right)\left(s_{2} r_{i j}^{\varepsilon_{2}} s_{2}^{-1}\right) \ldots\left(s_{m} r_{i j}^{\varepsilon_{m}} s_{m}^{-1}\right),
$$

где $s_{k}^{-1}=\operatorname{rev} s_{k}$ (то есть слово, записанное в обратном порядке), $k=1, \ldots, m$.

Так как $r_{i j}^{\varepsilon_{k}}$ начинается и заканчивается на разные буквы $a_{i}, a_{j}$, в множителях $s_{k} r_{i j}^{\varepsilon_{k}} s_{k}^{-1}$ идет сокращение до тех пор, пока $s_{k} r_{i j}^{\varepsilon_{k}} s_{k}^{-1}$ не станет равным $r_{i j}^{\varepsilon_{k}}$, либо $r_{j i}^{\varepsilon_{k}}, k=1, \ldots, m$. Таким образом, $R_{i j}=\left\{r_{i j}^{l}, r_{j i}^{m}\right\}, l, m \in \mathbf{N}$.

В дальнейшем под $R$ будем понимать

$$
R=\bigcup_{i, j \in J} R_{i j},
$$

симметризованное подмножество свободного произведения $F$. Тогда группа Кокстера может быть задана представлением $G=\left\langle A ; a_{i}^{2}, R\right\rangle, i=1, \ldots, n$. Пусть $w$ - нетривиальное циклически приведенное в $F$ слово, равное единице в группе $G$ Кокстера большого типа, то есть $w \in\langle R\rangle^{F}$, где $\langle R\rangle^{F}$ - нормальное замыкание симметризованного множества $R$ в свободном произведении $F$. Учитывая, что $a_{i}=a_{i}^{-1}$, в $F$ можно строить $R$-диаграммы над $F$ в точности так же, как над свободной группой (см. [3]). Следовательно, существует связная односвязная диаграмма $M$ группы Кокстера с граничной меткой $w$, областями которой являются $R_{i j}$-диаграммы с метками типа $(i, j)$.

Подвергнем $R$-диаграмму $M$ следующему преобразованию. Если две области $D_{1}, D_{2}$ являются одновременно $R_{i j}$-диаграммами, пересекаются по ребру с меткой $\varphi\left(\partial D_{1} \cap \partial D_{2}\right)$, то, стирая это ребро, объединим $D_{1}, D_{2}$ в одну область $D$. При этом возможно, что метка границы полученной области равна единице в свободном произведении $F$. Тогда, удалив эту область, склеиваем ее границу. Таким образом, через конечное число шагов мы получим приведенную (в $F$ ) связную односвязную $R$-диаграмму $M$, инвариантную относительного рассмотренного преобразования с граничной меткой, равной $w$, причем, если две области $D^{\prime}, D^{\prime \prime}$ из $M$ пересекаются по ребру, то длина метки этого ребра равна единице и справедливо следующее утверждение. 
Лемма 4 ([1]). Каждая приведенная связная односвязная $R$-диаграмма $M$ группы Кокстера большого типа удовлетворяет условию С(6).

Относительно условия $C(6)$, см. [1].

Обозначим через $\partial M$ граничный цикл $M$. Область $D \subset M$ назовем граничной, если $\partial M \cap \partial D \neq \varnothing$. Символом $i(D)$ будем обозначать число внутренних ребер в граничном цикле $D$.

Будем говорить, что $\partial D \cap \partial M$ есть правильная часть $M$, если $\partial D \cap \partial M$ есть объединение последовательности $l_{1}, l_{2}, \ldots, l_{n}$ замкнутых ребер, где $l_{1}, \ldots l_{n}$ встречаются в данном порядке в некотором граничном цикле для $D$ и в некотором граничном цикле для $M$.

Граничную область $D R$-диаграммы $M$ назовем простой, если $\partial D \cap \partial M$ есть правильная часть. Простая область $D$ диаграммы $M$ называется деновской, если $i(D)<3$.

Пусть $M$ - приведенная связная односвязная $R$-диаграмма группы Кокстера большого типа. Тогда последовательность граничных областей $D_{1}, D_{2}, \ldots, D_{n}, n \geqslant 2$, образует полосу в $M$, если

- $\partial D_{i} \cap \partial M-$ правильная часть $M$ для всех $i, 1 \leqslant i \leqslant n$;

- для всех $i, 1 \leqslant i<n$, границы областей $D_{i}$ и $D_{i+1}$ пересекаются по ребру;

- $i\left(D_{1}\right)=i\left(D_{n}\right)=3$ и для всех $j, 1<j<n, i\left(D_{j}\right)=4$.

Лемма 5 ([2]). Если $M$ - приведенная связная односвязная диаграмма типа C(6), не содержащая деновской области, то она содержит по крайней мере три непересекающиеся полосы.

Область $D$ с граничным циклом $\partial D=e \gamma e^{-1} \delta$, расположенная по обе стороны относительно ребра $e$, в которой склеенные ребра $e$ и $e^{-1}$ пересекают граничный цикл $D$, называется $(s-i)$-областью.

Лемма 6 ([3]). Пусть $M$ - приведенная связная диаграмма $M$ группы Кокстера большого типа, содержашая $(s-i)$-области, тогда $\varphi(j)$ и $\varphi(\delta)$ содержат одну букву.

Лемма 7. Если $M$ - приведенная связная односвязная $R$-диаграмма, то она не содержст $(s-i)$-областей.

Доказательство очевидно.

Удаление деновской области диаграммы $M$, то есть удаление ее граничного пути, называется деновским сокрашением диаграммы $M$ или $R$-сокращением. Будем говорить, что $M$ является $R$-приведенной, если она не содержит деновских областей.

Пусть $\Pi$ - полоса диаграммы $M, \partial M=\gamma \cup(\partial \Pi \cap \partial M), \gamma_{1}=\partial \Pi \backslash(\partial \Pi \cap \partial M)$. Замену диаграммы $M$ на диаграмму $M_{1}$, полученную из $M$ удалением полосы П, в результате чего граничный цикл $M$ преобразуется в граничный цикл $\partial M_{1}=\gamma \gamma_{1}$, назовем специальным $R$-сокращением или $\bar{R}$-сокращением. Если $M$ не содержит полос, то назовем $M$ специально $R$-приведенной или $\bar{R}$-приведенной. Слово $w \in G$, где $G$ - группа Кокстера большого типа, назовем $R$-приводимым, если $w$ приводимо в $F$ и содержит подслово $s$, являющееся подсловом некоторого соотношения $r \in R, r=s b$, где $|b| \leqslant 2$. Назовем $w$ циклически $R$-приведенным, если все его циклические перестановки являются $R$-приведенными словами. Если $w^{\prime}$ получено из $w R$-приведением, то $\left|w^{\prime}\right|<|w|$ на основании леммы 2 . 
Циклически $R$-приведенное слово $w$ группы $G$ Кокстера большого типа назовем специально $R$-приводимым, если в некоторой его циклической перестановке можно выделить подслово $s_{1} s_{2} \ldots s_{n}$, где каждое $s_{t}$ содержится в некоторой группе $G_{i j}$ и является подсловом соотношения $s_{t} d_{t}^{-1} b_{t} d_{t+1} \in R$, причем при $t=1$ и $t=n$

$$
\left|d_{1}\right|=\left|b_{1}\right|=\left|d_{2}\right|=\left|d_{n}\right|=\left|b_{n}\right|=\left|d_{n+1}\right|=1
$$

и для $t, 1<t<n$,

$$
\left|d_{t}\right|=\left|d_{t+1}\right|=1, \quad\left|b_{t}\right|=2 .
$$

Лемма 8. Пусть $w \in G, G$ - группа Кокстера большого типа, $w$ ииклически $R$-приведено. Тогда, если слово $w^{\prime}$ получено из $w$ спечиальным $R$-приведением, то $\left|w^{\prime}\right|<|w|$.

Доказательство очевидно.

Теорема 2. В группе $G_{i j}$ разрешима проблема равенства слов.

Доказательство непосредственно следует из предыдущих утверждений.

Лемма 9 ([2]). Существует алгоритм, позволяющий для любого чиклически приведенного слова ш группы Кокстера большого типа выяснить, является ли $w$ R-nриведенным.

Лемма 10 ([2]). Существует алгоритм, позволяючий для любого ииклически $R$-приведенного слова $ш$ из группь Кокстера большого типа выяснить, является ли $w$ специально $R$-приведенньм.

Теорема 3. В группах Кокстера большого типа разрешима проблема равенства слов.

Доказательство очевидно.

Приведенную связную односвязную $R$-диаграмму $M$ назовем чечевичной, если $M$ состоит из областей $D_{1}, \ldots, D_{n}$ таких, что для любого $i, 1 \leqslant i<n, D_{i} \cap D_{i+1}$ есть ребро, для любого $i, 1 \leqslant i \leqslant n, D_{i} \cap \gamma_{1} \neq \varnothing$ и $D_{i} \cap \gamma_{2} \neq \varnothing$, где $\gamma_{1} \gamma_{2}^{-1}$ есть граничный цикл $M$.

Пусть $M$ - приведенная связная односвязная $R$-диаграмма, каждая область которой не является правильной. Тогда $M$ состоит из чечевичных поддиаграмм, являющихся компонентами данной диаграммы, причем, если каждой компоненте поставить в соответствие точку, то получим граф, являющийся деревом. В таких диаграммах можно выполнить деновские сокращения.

Рассмотрим теперь кольцевые диаграммы над группами Кокстера большого типа. Назовем связную карту $M$ на плоскости $E^{2}$ кольцевой картой, если дополнение ее состоит в точности из двух компонент. Через $K$ обозначим неограниченную, а через $H$ - ограниченную компоненты множества $M$. Назовем $\partial M \cap \partial K$ внешней, а $\partial M \cap \partial H$ внутренней границей карты $M$. Цикл минимальной длины, содержащий все ребра внешней границы карты $M$, есть внешний граничный цикл карты $M$, обозначим его $\sigma$. Аналогично определяется внутренний граничный цикл карты $M$. Обозначим его $\tau$.

Пусть $F$ - свободное произведение, $R$ - симметризованное подмножество его элементов, $N$ - нормальное замыкание $R$ в $F$. Пусть $u$ и $z$ - два циклически приведенных слова из $F$, не лежащих в $N$ и не сопряженных в $F$. Если $u$ и $z$ представляют собой некоторые сопряженные элементы группы $G=F / N$, то существует приведенная кольцевая $R$-диаграмма $M$, содержащая не менее одной области, такая, что если $\sigma=e_{1} \ldots e_{s}$ и $\tau=t_{1} \ldots t_{k}-$ соответственно внутренний и внешний граничные циклы карты $M$, то 
произведение $\varphi\left(e_{1}\right) \ldots \varphi\left(e_{s}\right)$ циклически приведено и сопряжено элементу $u$ в $F$, а произведение $\varphi\left(t_{1}\right) \ldots \varphi\left(t_{k}\right)$ тоже циклически приведено и сопряжено $z^{-1}$ в $F$, где $z^{-1}=\operatorname{rev}(z)$ (см. [3]). Пусть $u, z$ - два циклически приведенных слова, $u, z \notin\langle R\rangle^{G}$, не сопряжены в $F$ и сопряжены в $G$. Тогда существует связная приведенная кольцевая $R$-диаграмма $M$ с внешней граничной меткой $u$ и внутренней граничной меткой $z^{-1}$, граничными метками областей $D$ которой являются соотношения из $R$. Подвергнем кольцевую $R$-диаграмму следующему преобразованию. Если две области $D_{1}, D_{2}$ пересекаются по ребру $e$, причем обшей граничной меткой является либо соотношение из некоторого симметризованного множества $R_{i j}$, либо слово, равное 1 в $F_{i j}$, то стерев ребро $e$, объединяем $D_{1}$ и $D_{2}$ в одну область, причем в случае равенства метки 1 в $F_{i j}$, данную область выбрасываем, а ее границу склеиваем. В результате получаем кольцевую $R$-диаграмму $M^{\prime}$ с условием $C(6)$ с теми же граничными метками $u$ и $z^{-1}$. В дальнейшем будем рассматривать кольцевые связные приведенные $R$-диаграммы $M$ для элементов группы Кокстера большого типа, инвариантные относительно указанных выше преобразований. Будем предполагать также, что $u$ и $z^{-1}$ являются циклически $R$-приведенными и специально $R$-приведенными словами. Заметим, что меткой каждого внутреннего ребра будет буква $a_{j} \in A$.

Изучим кольцевые $R$-диаграммы $M$ для элементов групп Кокстера большого типа, не содержащих $(s-i)$-областей.

Лемма 11. Пусть $M$ - связная приведенная кольцевая $R$-диаграмма с граничными метками $\varphi(\sigma), \varphi(\tau)$, принадлежащими группе Кокстера большого типа, $\varphi(\sigma), \varphi(\tau)-$ спечиально $R$-приведенные слова. Тогда, если каждая граничная область $D$ карты $M$ пересекается только с внешним, либо только с внутренним граничным чиклом $M$, то дM $\cap \partial$ - связное множество.

Доказательство очевидно.

Обозначим $\sum^{*}$ символ суммирования по граничным областям диаграммы.

Лемма 12. Пусть $M$ - кольчевая связная приведенная $R$-диаграмма с условием $C(6)$, каждая граничная область $D$ которой такова, что дD $\cap 2 M-$ связное множество. Tогдa

$$
\sum_{M}^{*}(4-i(D)) \geqslant 0,
$$

где суммирование проводится по всем граничным областям диаграммы $M u i(D)-$ степень области $D$.

Доказательство. Напомним (см. [5]), что [p, q]-картой называется карта, все внутренние вершины $v$ которой имеют степень $d(v)$ не менее $p$, а все (внутренние) области $D$ имеют степень $i(D)$ не менее $q$. Для кольцевых связных $[p, q]$-карт справедлива формула (см. [5])

$$
\sum_{M}^{*}(p / q+2-d(v)) \geqslant 0
$$

где суммирование проводится по всем вершинам $v \in M$. Карты $C(6)$ есть $[3,6]$-карты (карты с условием $(3,6)$ ). Пусть $M$ - кольцевая карта с условием $(3,6)$, тогда $M^{*}$, дуальная к ней карта, будет [6, 3]-картой, для которой имеет место соотношение

$$
\sum_{M^{*}}^{*}\left(4-d\left(v^{*}\right)\right) \geqslant 0,
$$


где суммирование проводится по граничным вершинам $v^{*}$ карты $M^{*}$, каждая из которых соответствует граничной области $D$ карты $M$. В силу дуальности $M^{*}$ и $M$ для указанных вершин $v^{*}$ и областей $D$ справедливо равенство $d\left(v^{*}\right)=i(D)$.

Пусть $M$ - связная кольцевая $R$-диаграмма, для каждой граничной области $D$ которой $\partial D \cap \partial M-$ связное множество. Тогда внешний (внутренний) граничный слой $M$ образует кольцевую связную $R$-диаграмму, которую будем называть внешним (внутренним) $K$-слоем диаграммы $M$ и обозначать соответственно через $K_{\sigma}\left(K_{\tau}\right)$, где $\sigma(\tau)-$ соответственно внешний (внутренний) граничный циклы диаграммы $M$.

Пусть $M$ - связная кольцевая $R$-диаграмма, являющаяся диаграммой сопряженности слов из группы Кокстера большого типа. Тогда внешний (внутренний) $K$-слой из $M$ назовем специальным внешним (внутренним) $K$-слоем, если образующие его области $D_{1}, \ldots, D_{n}$, удовлетворяют следующим условиям:

(1) $D_{j} \cap D_{j+1}$ есть ребро для всех $j, 1 \leqslant j<n-1$,

(2) $D_{1} \cap D_{n}$ есть ребро,

(3) $i\left(D_{1}\right)=3$,

(4) $i\left(D_{2}\right)=i\left(D_{3}\right)=\ldots=i\left(D_{n}\right)=4$.

Пусть $M$ - кпльцевая связная приведенная $R$-диаграмма с условием $C(6)$ над группами Кокстера большого типа. Обозначим через $M^{\prime}$ кольцевую диаграмму, полученную из $M$ удалением внешнего граничного слоя $K_{\sigma}$, через $M^{\prime \prime}$ - диаграмму, полученную из $M$ удалением внутреннего граничного слоя $K_{\tau}$, а через $M^{\prime \prime \prime}$ - диаграмму, полученную из $M$ удалением внутреннего и внешнего граничного слоев.

Лемма 13. Пусть $M-$ связная кольчевая приведенная $R$-диаграмма сопряженности слов из группь $G$ Кокстера большого типа, у которой $\sigma$ - внешний, $\tau$ - внутренний граничный чиклы соответственно с метками $\varphi(\sigma), \varphi(\tau)$, являющимися чиклически $R$-приведенными словами. Пусть также каждая граничная область $D$ карты $M$ пересекается либо с $\sigma$, либо с $\tau$. Тогда если $K$ - спечиальный внешний (внутренний) слой $M$, внешней и внутренней граничной меткой которого соответственно являются слова $w$, $v$ из $G$, mo $|v|<|w|$.

Используя непосредственно структуру специального слоя и леммы 1 и 2, получаем необходимую оценку.

Лемма 14. Пусть $M-$ связная приведенная кольцевая $R$-диаграмма сопряженности элементов из группы Кокстера большого типа, $\sigma, \tau$-соответственно внешний и внутренний граничные чиклы $M$, метки которых $\varphi(\sigma), \varphi(\tau)$ спечиально $R$-приведены, и пусть каждая граничная область $D$ из $M$ пересекается либо с $\sigma$, либо $c \tau$. Тогда если внешний и внутренний слои карты $M$ не являются спещиальными, то

$$
\sum_{\sigma}^{*}(4-i(D)) \geqslant 0, \quad \sum_{\tau}^{*}(4-i(D)) \geqslant 0 .
$$

Доказательство. Допустим, что утверждение леммы не является справедливым, то есть, например, $\sum_{\sigma}^{*}(4-i(D))<0$. Тогда существует натуральное число $k_{0}$ такое, что

$$
\sum_{\sigma}^{*}(4-i(D))+k_{0}=0 .
$$


В силу леммы 12

$$
\sum_{M}^{*}(4-i(D))=\sum_{\sigma}^{*}(4-i(D))+\sum_{\tau}^{*}(4-i(D)) \geqslant 0,
$$

поэтому $\sum_{\tau}^{*}(4-i(D))>0$. Пусть $D_{j_{1}}, D_{j_{2}}, \ldots, D_{j_{m}}-$ граничные области $M$, принадлежащие внутреннему граничному слою $M$, каждая из которых дает отрицательный вклад в общую сумму $\sum_{\tau}^{*}(4-i(D))$, то есть

$$
\sum_{s=1}^{m}\left(4-i\left(D_{j s}\right)\right)=k
$$

где $k<0$. Для того чтобы для карты $M$ выполнялись соотношения

$$
\sum_{\tau}^{*}(4-i(D))>0, \quad \sum_{M}^{*}(4-i(D)) \geqslant 0,
$$

необходимо, чтобы внутренний граничный слой содержал, по меньшей мере, $|k|+k_{0}$ областей с внутренней степенью, равной трем. Отсюда следует, что либо вдоль границы $\tau$ можно выделить полосу, а следовательно, метка $\varphi(\tau)$ специально $R$-приводима, либо внутренний граничный слой карты $M$ является специальным.

Пусть $M$ - связная карта, состоящая из связной кольцевой диаграммы $M_{1}$ и связной односвязной диаграммы $M_{2}$, соединенных друг с другом некоторым простым путем, возможно, нулевой длины. Назовем диаграмму $M_{2}$ островом.

Если связная кольцевая диаграмма $M_{1}$ связной карты $M$ соединена со связной односвязной диаграммой $M_{2}$ посредством некоторой области $D$, где $\partial D \cap \partial M-$ несвязное множество и внутренняя степень области $D$ относительно каждой диаграммы $M_{i}$, $i=1, \ldots, 2$, не меньше единицы, то диаграмму $M_{2}$ назовем полуостровом $M$.

Лемма 15 ([2]). Пусть $M-$ приведені:ая связная кольчевая $R$-диаграмма сопряженности слов $\varphi(\sigma), \varphi(\tau) \in G, G$ - группа Кокстера большого типа, $\sigma, \tau$ - соответственно внешний и внутренний граничный чикль $M$, каждая граничная область $D$ из $M$ пересекается либо с $\sigma$, либо с $\tau$, слова $\varphi(\sigma), \varphi(\tau)$ спечиально $R$-приведены. Тогда диаграмма $M^{\prime}\left(M^{\prime \prime}\right)$, полученная из $M$ удалением внешнего $K_{\sigma}$ (внутреннего $K_{\tau}$ ) граничного слоя $M$, не содержит ни островов, ни полуостровов.

Пусть $M$ - приведенная связная кольцевая $R$-диаграмма сопряженности слов $\varphi(\sigma)$, $\varphi(\tau)$ группы Кокстера большого типа, $\sigma, \tau$ - соответственно внешний и внутренний граничный циклы $M$, каждая граничная область $D$ пересекается либо с $\sigma$, либо с $\tau$, слова $\varphi(\sigma), \varphi(\tau)$ специально $R$-приведены и

$$
\sum_{\sigma}^{*}(4-i(D))=0 \quad\left(\sum_{\tau}^{*}(4-i(D))=0\right) .
$$

Тогда $M$ назовем специальной кольцевой $R$-диаграммой.

Лемма 16 ([2]). Пусть $M$ - спечиальная кольчевая $R$-диаграмма. Тогда внешний (внутренний) граничный $K$-слой карты $M$ не содержит области $D$ c $i(D)>5$ и в $К$ число областей с внутренней степенью 3 равно числу областей с внутренней степенью 5. 
Лемма 17. Пусть $M$ - приведенная связная кольчевая $R$-диаграмма сопряженности слов $\varphi(\sigma), \varphi(\tau) \in G, G$ - группа Кокстера большого типа, $\sigma, \tau$ - соответственно внешний и внутренний граничный чиклы $M$, каждая граничная область $D$ пересекается либо с б, либо с $\tau$, слова $\varphi(\sigma), \varphi(\tau)$ спечиально $R$-приведены. Тогда если внешний и внутренний граничные слои карты $M$ не являются спечиальными, то

$$
\sum_{\sigma}^{*}(4-i(D))=0, \quad \sum_{\tau}^{*}(4-i(D))=0 .
$$

Доказательство очевидно.

Кольцевую связную приведенную $R$-диаграмму $M$ сопряженности слов $\varphi(\sigma)$, $\varphi(\tau) \in G$, где $\sigma, \tau$ - соответственно внешний и внутренний граничный циклы $M$, назовем минимальной, если не существует кольцевой $R$-диаграммы $M_{0}$ с теми же граничными метками $\varphi(\sigma), \varphi(\tau)$, имеющей меньшее число областей. Очевидно, что минимальная кольцевая связная $R$-диаграмма сопряженности слов $\varphi(\sigma)$ и $\varphi(\tau)$ приведена.

Лемма 18. Пусть $M$ - спечиальная кольчевая $R$-диаграмма группы $G$ Кокстера большого типа с граничными чиклами $\sigma$, $\tau$. Тогда

$$
\left|\varphi\left(\partial K_{\sigma} \cap \partial M\right)\right| \geqslant\left|\varphi\left(\partial K_{\sigma} \cap \partial M^{\prime}\right)\right| \quad\left(\left|\varphi\left(\partial K_{\tau} \cap \partial M\right)\right| \geqslant\left|\varphi\left(\partial K_{\tau} \cap \partial M^{\prime \prime}\right)\right|\right),
$$

где $K_{\sigma}-$ внешний $\left(K_{\tau}-\right.$ внутренний) граничный слой $M$.

Доказательство. Из леммы 16 следует, что граничный слой $K_{\sigma}\left(K_{\tau}\right)$ не содержит областей $D$ с $i(D)>5$ в $M$ и в $K_{\sigma}\left(K_{\tau}\right)$, число областей с внутренней степенью 5 равно числу областей с внутренней степенью 3, причем любые две области с внутренней степенью 5 разделены областью с внутренней степенью 3 , остальные области из $K_{\sigma}\left(K_{\tau}\right)$ имеют внутреннюю степень 4. Разобьем последовательность областей из $K_{\sigma}$ на последовательности следующим образом:

$$
\begin{aligned}
D_{1}, D_{2}, \ldots, D_{j_{1}}, D_{j_{1}+1}, \ldots, D_{k_{1}} ; D_{k_{1}+1}, \ldots, D_{k_{1}+j_{2}}, \ldots, D_{k_{2}}, \ldots ; \\
D_{k_{i}+1}, \ldots, D_{k_{i}+j_{i+1}}, \ldots, D_{k_{i+1}}, \ldots ; D_{k_{r}+1}, \ldots, D_{k_{r}+j_{r+1}}, \ldots, D_{k_{r+1}},
\end{aligned}
$$

где

$$
\begin{array}{r}
i\left(D_{1}\right)=i\left(D_{k_{1}+1}\right)=\ldots=i\left(D_{k_{i}+1}\right)=\ldots=i\left(D_{k_{r}+1}\right)=5, \\
i\left(D_{j_{1}}\right)=i\left(D_{k_{1}+j_{2}}\right)=\ldots=i\left(D_{k_{i}+j_{i+1}}\right)=\ldots=i\left(D_{k_{r}+j_{r+1}}\right)=3 .
\end{array}
$$

Все остальные области имеют внутреннюю степень 4 . Заметим, что в $K_{\sigma}$ за областью $D_{k_{r+1}}$ следует область $D_{1}$. Рассмотрим подкарту $K_{\sigma}$-слоя, образованную областями $D_{k_{i}+1}, \ldots, D_{k_{i}+j_{i+1}}, \ldots, D_{k_{i+1}}$, при $i=0, k_{0}=0$. Обозначим метку произвольной области $D_{k_{i+t}}, 1 \leqslant t \leqslant k_{i+1}-k_{i}$, следующим образом:

$$
\varphi\left(\partial D_{k_{i+t}}\right)=s_{k_{i+t}}^{-1} d_{k_{i+t}}^{-1} b_{k_{i+t}} d_{k_{i+t+1}}^{-1}
$$

Из сказанного выше следует, что

$$
\left|h_{k_{i+1}}\right|=3, \quad\left|h_{k_{i+j_{i}+1}}\right|=1, \quad\left|h_{k_{i+t}}\right|=2, \quad 2 \leqslant t<j_{i+1}, \quad j_{i+1}<t \leqslant k_{i+1}, \quad\left|d_{s}\right|=1
$$


для любого $s$. Используя леммы 1 и 2, получаем, что

$$
\begin{aligned}
\left|b_{k_{i+1}}\right| & \leqslant\left|d_{k_{i+1}}\right|+\left|s_{k_{i+1}}\right|+\left|d_{k_{i+2}}\right|, \\
\left|b_{k_{i+2}}\right| & +\left|d_{k_{i+2}}\right| \leqslant\left|d_{k_{i+3}}\right|+\left|s_{k_{i+2}}\right|, \ldots,\left|b_{k_{i}+j_{i+1}-1}\right|+\left|d_{k_{i}+j_{i+1}-1}\right| \\
& \leqslant\left|d_{k_{i}+j_{i+1}}\right|+\left|s_{k_{i}+j_{i+1}-1}\right|, \\
\left|d_{k_{i}+j_{i+1}}\right| & +\left|b_{k_{i}+j_{i}-1}\right|+\left|d_{k_{i}+j_{i+1}+1}\right| \leqslant\left|s_{k_{i}+j_{i}+1}\right|, \\
\left|b_{k_{i}+j_{i+1}+1}\right| & +\left|d_{k_{i}+j_{i+1}+2}\right| \leqslant\left|s_{k_{i}+j_{i+1}+1}\right|+\left|d_{k_{i}+j_{i+1}+1}\right|, \ldots,\left|b_{k_{i}+1}\right|+\left|d_{k_{i+1}+1}\right| \\
& \leqslant\left|s_{k_{i+1}}\right|+\left|d_{k_{i+1}}\right| .
\end{aligned}
$$

Суммируя отдельно левые и правые части неравенств, получаем неравенство

$$
\sum_{t=1}^{k_{i+1}-k_{i}}\left|b_{k_{i+t}}\right|+\left|d_{k_{i+1}+1}\right| \leqslant \sum_{t=1}^{k_{i+1}-k_{i}}\left|s_{k_{i+t}}\right|+\left|d_{k_{i+1}}\right| .
$$

Учитывая, что $d_{1}=d_{r+1}+1$, неравегство

$$
\sum_{i=0}^{r}\left(\sum_{t=1}^{k_{i+1}-k_{i}}\left|b_{k_{i+t}}\right|+\left|d_{k_{i+1}+1}\right|\right) \leqslant \sum_{i=0}^{r}\left(\sum_{t=1}^{k_{i+1}-k_{i}}\left|s_{k_{i+t}}\right|+\left|d_{k_{i+1}}\right|\right)
$$

преобразуем к виду

$$
\sum_{i=1}^{k_{r+1}}\left|b_{i}\right| \leqslant \sum_{i=1}^{k_{r+1}}\left|s_{i}\right|
$$

Из последнего неравенства следует, что

$$
\left|\varphi\left(\partial K_{\sigma} \cap \partial M^{\prime}\right)\right| \leqslant\left|\varphi\left(\partial K_{\sigma} \cap \partial M\right)\right| .
$$

Кольцевую связную приведенную однослойную $R$-диаграмму $M$ с граничными циклами $\sigma, \tau$ группы Кокстера большого типа, метки которой $\varphi(\sigma), \varphi(\tau)$ приведены в $F, \varphi(\sigma)$ $R$-приведено и специально $R$-приведено, назовем особо специальной $R$-диаграммой, если в $M$ существует одна область $D$ такая, что

$$
|\varphi(\partial D \backslash(\partial D \cap \sigma))|=3,
$$

а для остальных областей $D^{\prime}$

$$
\left|\varphi\left(\partial D^{\prime} \backslash\left(\partial D^{\prime} \cap \sigma\right)\right)\right|=4
$$

Слово $\varphi(\tau)$ является циклически $R$-приведенным и циклически специально $R$-приведенным. Замену слова $\varphi(\sigma)$ словом $\varphi(\tau)$ назовем специальным кольцевым $R$-сокращением.

Лемма 19 ([2]). Существует алгоритм, позволяющий для любого чиклически $R$-приведенного чиклически спечиально $R$-приведенного слова w из группы Кокстера большого типа установить, применимо ли к нему спечиальное кольчевое $R$-сокращение.

Рассмотрим кольцевые связные приведенные $R$-диаграммы $M$ сопряженности слов групп Кокстера большого типа с граничными циклами $\sigma, \tau$, у которых не каждая граничная область явлется простой. При этом кольцевая $R$-диаграмма $M$ может быть одного из следующих видов: 
$\left(1^{\circ}\right) \sigma \cap \tau=\varnothing$, каждая область $D \in M$ граничная, $\partial D \cap \partial M$ - несвязное множество, $i(D)=2$;

$\left(2^{\circ}\right) \sigma \cap \tau \neq \varnothing$.

$\left(3^{\circ}\right) \sigma \cap \tau=\varnothing$, существует область $D$ такая, что $i(D)>2, \partial D \cap \partial M-$ несвязное множество.

Будем говорить, что циклически несократимое слово $w$ группы Кокстера большого типа обладает свойством $s$, если $w$ циклически $R$-несократимо, циклически специально $R$-несократимо и к нему неприменимо кольцевое специальное $R$-сокращение.

Лемма 20. Пусть $u, v$ - слова группы $G$ Кокстера большого типа, обладающие свойством $s$. Тогда можно эффективно установить, являются ли слова $u, v$ граничными метками кольчевой связной приведенной $R$-диаграммы вида $2^{\circ}$.

Доказательство очевидно.

Лемма 21. Пусть $u, v$ - слова группы Кокстера большого типа, обладающие свойством $s$. Тогда если $u, v$ - граничные метки кольцевой связной приведенной $R$-диаграммы $M$ вида $3^{\circ}$ с граничными циклами $\sigma, \tau, \varphi(\sigma)=u, \varphi(\tau)=v^{-1}$, то существует простой путь $\chi=e_{1} e_{2}$, принадлежащий граничному чиклу некоторой области $D$, соединяющей граничные вершины из $\sigma$ и $\tau$, метка которого может быть эффективно определена.

Доказательство. Кольцевая $R$-диаграмма $M$ с условием $C(6)$ с граничными метками $\varphi(\sigma)=u, \varphi(\tau)=v$, удовлетворяющими условиям леммы, является специальной $(C-1)-$ слойной диаграммой [6].

Из лемм 17, 19, 22 статьи [6] следует, что диаграмма $M$ состоит из связных односвязных диаграмм $\Gamma_{i}, i=1, \ldots, p$, каждая область $D$ которых, граничная в $M$, является простой и каждая $\Gamma_{i}$ в $M$ ограничена слева и справа соответственно областями $\widetilde{D}_{i}, \widetilde{D}_{i}^{\prime}$, причем $\partial \widetilde{D}_{i} \cap \partial M$ и $\partial \widetilde{D}_{i}^{\prime} \cap \partial M-$ несвязные множества,

$$
\begin{aligned}
& \partial \tilde{D}_{i} \cap \partial \Gamma_{i}=c_{i-1}, \quad \partial \tilde{D}_{i}^{\prime} \cap \partial \Gamma_{i}=c_{i}, \quad\left|c_{i-1}\right|=\left|c_{i}\right|=2, \\
& \partial \Gamma_{i}=\gamma_{i} c_{i-1} \delta_{i} c_{i}, \quad \partial \Gamma_{i} \cap \sigma=\gamma_{i}, \quad \partial \Gamma_{i} \cap \tau=\delta_{i},
\end{aligned}
$$

откуда следует существование простого пути $\chi$.

Кроме того, в лемме 22 из [6] показано, что среди областей

$$
K_{\sigma} \cap \Gamma_{i}=\left\{D_{1}, D_{2}, \ldots, D_{m}\right\}, \quad\left(K_{\tau} \cap \Gamma_{i}=\left\{D_{1}^{\prime}, D_{2}^{\prime}, \ldots, D_{n}^{\prime}\right\}\right),
$$

где

$$
\partial D_{1} \cap c_{i-1}=e_{2}^{\prime}, \quad \partial D_{1}^{\prime} \cap c_{i-1}=e_{1}^{\prime}, \quad \partial D_{m} \cap c_{i}=e_{2}^{\prime \prime}, \quad \partial D_{n}^{\prime} \cap c_{i}=e_{1}^{\prime \prime},
$$

содержится область $D_{k}, 1 \leqslant k \leqslant m,\left(D_{t}^{\prime}, 1 \leqslant t \leqslant n\right)$ такая, что

$$
i\left(D_{k}\right)=3, \quad i\left(D_{1}\right)=\ldots=i\left(D_{k-1}\right)=4 \quad\left(i\left(D_{t}^{\prime}\right)=3, \quad i\left(D_{1}^{\prime}\right)=\ldots=i\left(D_{t-1}^{\prime}\right)=4\right) .
$$

Теперь построим алгоритм, с помощью которого можно выяснить, существует ли слово $\varphi(\chi) \in G$ такое, что $\varphi(\chi)^{-1} u^{*} \varphi(\chi)=v^{*}$, где $u^{*}, v^{*}$ - некоторые циклические перестановки $u, v ; \varphi(\chi)$ - метка пути $\chi=e_{1}^{\prime} e_{2}^{\prime}=c_{i-1}$, соединяющего некоторые вершины из $\sigma$ и $\tau, \chi=c_{i-1}$ принадлежит граничному циклу некоторой области $D_{i-1}^{\prime}$. Разобьем циклическое слово $u(v)$ на подслова $u_{1}, u_{2}, \ldots, u_{m}\left(v_{1}, v_{2}, \ldots, v_{n}\right)$, где $u_{s} \in G_{i_{s} j_{s}}, s=1, \ldots, m$ $\left(v_{t} \in G_{i_{t} j_{t}}, t=1, \ldots, n\right)$. 
Подслова $u_{i}, i=1, \ldots, m\left(v_{t}, t=1, \ldots, n\right)$ занумеруем в направлении обхода $\sigma(\tau)$ по часовой стрелке. Данному разбиению $u(v)$ соответствует разбиение границы $\sigma(\tau)$ на простые пути $\sigma_{1}, \sigma_{2}, \ldots, \sigma_{m}\left(\tau_{1}, \tau_{2}, \ldots, \tau_{n}\right)$, где $\varphi\left(\sigma_{i}\right)=u_{i}, i=1, \ldots, m\left(\varphi\left(\tau_{j}\right)=v_{j}\right.$, $j=1, \ldots, n)$. Соединим правый конец $\sigma_{1}$ с правым концом какого-нибудь $\tau_{j}$, например с $\tau_{1}$, отрезком $\chi$; отметим, что $\varphi\left(\sigma_{1}\right)$ и $\varphi\left(\tau_{1}\right)$ должны содержаться в одной подгруппе $G_{i j}$. Разбиваем $\chi$ на два ребра $e_{1}^{\prime}, e_{2}^{\prime}, \chi=e_{1}^{\prime} e_{2}^{\prime}$, в направлении от $\tau$ к $\sigma$. Укажем способ нахождения меток $\varphi\left(e_{1}\right), \varphi\left(e_{2}\right)$. Для этого к каждому пути $\sigma_{2}, \ldots, \sigma_{k}, 2 \leqslant k \leqslant m$ $\left(\tau_{2}, \ldots, \tau_{t}, 2 \leqslant t \leqslant n\right)$ пристраиваем области $D_{2}, \ldots, D_{k}\left(D_{2}^{\prime}, \ldots, D_{t}^{\prime}\right)$, удовлетворяющие условиям, состоящим в том, что $\partial D_{2} \cap \chi=e_{2}^{\prime}\left(\partial D_{2}^{\prime} \cap \chi=e_{1}^{\prime}\right)$ и $D_{i}, D_{i+1}, i=2, \ldots, k-1$ $\left(D_{j}^{\prime}, D_{j+1}^{\prime}, j=2, \ldots, t-1\right)$ пересекаются по ребру; $\left|\varphi\left(\partial D_{i} \backslash \partial D_{i} \cap \sigma\right)\right|=4$, если $2 \leqslant i<k$; $\left|\varphi\left(\partial D_{j}^{\prime} \backslash \partial D_{j}^{\prime} \cap \tau\right)\right|=4$, если $2 \leqslant j<t-1 ;\left|\varphi\left(\partial D_{k} \backslash \partial D_{k} \cap \sigma\right)\right|=3,\left|\varphi\left(\partial D_{t}^{\prime} \backslash \partial D_{t}^{\prime} \cap \tau\right)\right|=3$, причем процесс построения областей $D_{i}\left(D_{j}^{\prime}\right)$ начинается со случая, когда $k=2(t=2)$; алгоритм построения областей аналогичен указанному в лемме, за тем исключением, что всякий раз он начинается с нахождения областей $D_{k}\left(D_{t}^{\prime}\right)$.

Метка каждой области $D \subset M$ удовлетворяет условию $|\varphi(\partial D)| \geqslant 6$, поэтому

$$
\left|u_{i}\right| \leqslant 2, \quad i=2, \ldots, k-1, \quad\left|u_{k}\right| \geqslant 3 \quad\left(\left|v_{j}\right| \geqslant 2, \quad j=2, \ldots, t-1, \quad\left|v_{t}\right| \geqslant 3\right) .
$$

Если для данного разбиения циклических слов $u, v$ и фиксированного соединения концов $\sigma_{1}, \tau_{1}$ можно построить интересующие нас последовательности областей $\left\{D_{i}\right\}_{i=2, \ldots, k}$, $\left\{D_{j}^{\prime}\right\}_{j=2, \ldots, t}$, для некоторых $k$ и $t, 2 \leqslant k \leqslant m, 2 \leqslant t \leqslant n$, то тем самым будет определена метка $\varphi(\chi)$, для которой проверяется выполнимость равенства $\varphi(\chi)^{-1} u^{*} \varphi(\chi)=v^{*}\left(u^{*}\right.$, $v^{*}$ - циклические перестановки $\left.u, v\right)$ и существование области $\tilde{D}_{i}, \partial \widetilde{D}_{i}=\sigma_{1} c \tau_{1} \chi$, где $\varphi\left(\sigma_{1}\right)=u_{i}^{-1}, \varphi\left(\tau_{1}\right)=v_{1}, \varphi(\chi)=\varphi\left(e_{1}^{\prime}\right) \varphi\left(e_{2}^{\prime}\right)$ и $|\varphi(c)|$ согласно лемме 18 удовлетворяет либо условию $|\varphi(c)|=1$, либо условию $|\varphi(c)|=2$. Если одно из указанных выше условий не выполняется, то определяем метку пути $\chi$, соединяющего конец $\sigma_{1}$ с концом $\tau_{2}$, если $\varphi\left(\sigma_{1}\right), \varphi\left(\tau_{2}\right)$ содержатся в одной подгруппе $G_{i j}$.

Таким образом, применяя указанный процесс для различных соединений концов $\sigma_{i}$, $\tau_{j}$ отрезком $\chi$ и для различных разбиений слов $u, v$ на подслова, мы через конечное число шагов можем проверить, существует ли искомое $\varphi(\chi)$.

Лемма 22 ([2]). Пусть $u, v$ - слова группь Кокстера большого типа, обладающие свойством s. Тогда можно эффективно установить, являются ли и, v граничными метками кольчевой связной приведенной $R$-диаграммы $M$ вида $1^{\circ}$.

Пусть $w$ - произвольное слово группы Кокстера большого типа. Рассмотрим следующие преобразования, укорачивающие длину циклического слова $w$ :

$\left(\pi_{1}\right)$ циклическое сокращение $w$ в свободном произведение $F$;

$\left(\pi_{2}\right)$ циклическое $R$-сокращение в $G$;

$\left(\pi_{3}\right)$ циклическое специальное $R$-сокращение в $G$;

$\left(\pi_{4}\right)$ кольцевое специальное $R$-сокращение в $G$;

$\left(\pi_{5}\right)$ переход от $w$ к сопряженному слову $u,|u|<|w|$, с помощью кольцевой диаграммы вида $1^{\circ}$;

$\left(\pi_{6}\right)$ то же, что $\pi_{5}$, но с помощью кольцевой диаграммы вида $2^{\circ}$;

$\left(\pi_{7}\right)$ то же, что $\pi_{5}$, но с помощью кольцевой диаграммы вида $3^{\circ}$. 
Слово $w$, полученное из $v$ применением к нему преобразований $\pi_{1}-\pi_{7}$ в $G$, назовем тупиковым для $v$, если оно инвариантно относительно этих преобразований.

Из лемм 9-22 следует, что для любого слова $v \in G$ можно эффективно построить соответствующее ему тупиковое слово $w$.

Кольцевую связную приведенную $R$-диаграмму $M$ с граничными циклами $\sigma, \tau$ назовем простой, если $|M| \geqslant 1$ и $\sigma \cap \tau \neq \varnothing ; M$ назовем вырожденной, если $|M|=0$, где $|M|$ - число областей диаграммы $M$.

Кольцевая связная приведенная $R$-диаграмма $M$ с граничными циклами $\sigma, \tau$ называется $n$-слойной, $n \geqslant 1$, если после последовательного удаления граничных слоев получим вырожденную кольцевую $R$-диаграмму, и называется $(C-n)$-слойной, если в результате удаления указанных выше граничных слоев получим простую кольцевую $R$-диаграмму.

Лемма 23. Пусть $v \in G, G-$ группа Кокстера большого типа, $v$ обладает свойством $s$ $u w$ - тупиковое для $v$ слово. Тогда никакое слово $u \in G,|u|<|w|$, не сопряжено с $v$.

Доказательство непосредственно использует понятие тупикового слова, $R$-сокращения и специального $R$-сокращения.

Пусть $M$ - кольцевая специальная $R$-диаграмма типа $C(6)$ с граничными циклами $\sigma$, $\tau$. Тогда граничный слой $K_{\sigma}\left(K_{\tau}\right)$ назовем $R K$-слоем, если

$$
\left|\partial K_{\sigma} \cap \partial M\right|=\left|\partial K_{\sigma} \cap \partial M^{\prime}\right| \quad\left(\left|\partial K_{\sigma} \cap \partial M\right|=\left|\partial K_{\sigma} \cap \partial M^{\prime \prime}\right|\right),
$$

где $M^{\prime}\left(M^{\prime \prime}\right)$ получена из $M$ удалением внешнего $K_{\sigma}$ (внутреннего $K_{\tau}$ ) слоя.

Лемма 24. Пусть $M$ - кольчевая связная приведенная минимальная $R$-диаграмма вида $C(6)$ группы $G$ Кокстера большого типа с граничными циклами $\sigma, \tau$. Пусть $\varphi(\sigma), \varphi(\tau)$ являются тупиковыми. Если $M-n$-слойная либо $(C-n)$-слойная диаграмма с $n>1$, то $d(D)=6$ для любой области $D \subset M$.

Доказательство. Пусть $M-n$-слойная $R$-диаграмма, $\varphi(\sigma), \varphi(\tau)$ являются тупиковыми, тогда $M$ - специальная $R$-диаграмма, у которой $K_{\sigma}, K_{\tau}$ являются $R K$-слоями. Если $n=2$, то утверждение леммы очевидно. Если $n>2$, то $M^{\prime}$ и $M^{\prime \prime}$ являются специальными и в силу леммы 18 из [6] $K_{\sigma^{\prime}}$ и $K_{\tau^{\prime}}$ являются $R K$-слоями. Отсюда следует, что для $n$-слойной $R$-диаграммы лемма справедлива.

Пусть $M-(C-n)$-слойная $R$-диаграмма, являющаяся, как и в предыдущем случае, специальной, граничные слои которой $K_{\sigma}, K_{\tau}-R K$-слои. Рассмотрим $R$-диаграмму $\tilde{M}$, полученную из $M$ удалением простой кольцевой диаграммы $M_{n}, M_{n}$ имеет граничные циклы $\sigma_{n}, \tau$. Как доказано в лемме 22 из [6], в диаграмме $\tilde{M}$, являющейся $n$-слойной с граничными циклами $\sigma, \sigma_{n}$, слой $K_{\sigma_{n}}$ является $R K$-слоем, поэтому все области $D \subset \tilde{M}$ имеют $d(D)=6$. Покажем, что любая область $D \subset M_{n}$ имеет $d(D)=6$.

Если $M_{n} \subset K_{\tau}$, то лемма справедлива. Пусть $M_{n} \not \subset K_{\tau}$. Простая кольцевая диаграмма $M_{n}$ состоит из односвязных компонент $M_{n}^{(1)}, \ldots, M_{n}^{(p)}$, каждая из которых является поддиаграммой некоторой Г-диаграммы кольцевой $M_{n-1}$-диаграммы, полученной из $M=M_{0}$ удалением граничных слоев $K_{\sigma_{0}}, K_{\sigma_{1}}, \ldots, K_{\sigma_{n-2}}$. Пусть $M_{n}^{(s)}, 1 \leqslant s \leqslant p$, - произвольные компоненты $M_{n}$ и $\chi_{s}, \chi_{s}^{\prime}$ - простые пути, соединяющие $M_{n}^{(s)}$ с предыдущей и последующей компонентами $M_{n}, v_{s}=\chi_{s} \cap M_{n}^{(s)}, v_{s}^{\prime}=\chi_{s}^{\prime} \cap M_{n}^{(s)}, v_{s}, v_{s}^{\prime}-$ вершины.

Из структуры диаграммы $\Gamma$, в которой содержится $M_{n}^{(s)}$ следует, что в $M_{n}^{(s)}$ содержатся области $D, D^{\prime}$, для которых

$$
\partial D=p_{1} p_{2} p_{3}, \quad \partial D \cap \sigma_{n}=p_{1}, \quad \partial D \cap \tau=p_{2}, \quad 1 \leqslant\left|p_{3}\right| \leqslant 2, \quad p_{1} \cap p_{2}=v_{s},
$$


аналогично,

$$
\partial D^{\prime}=p_{3}^{\prime} p_{2}^{\prime} p_{1}^{\prime}, \quad p_{2}^{\prime}=\partial D^{\prime} \cap \tau, \quad p_{1}^{\prime}=\partial D^{\prime} \cap \sigma_{n}, \quad 1 \leqslant\left|p_{3}^{\prime}\right| \leqslant 2, \quad p_{1}^{\prime} \cap p_{2}^{\prime}=v_{s}^{\prime} .
$$

Выделим в $M_{n}$ все поддиаграммы $\Gamma_{1}, \Gamma_{2}, \ldots, \Gamma_{t}$ типа $\Gamma$, каждая $\Gamma_{i}$ ограничена слева областью $D_{i}$, справа областью $D_{i}^{\prime}, D_{i}, D_{i}^{\prime} \subset K_{\tau}$,

$$
\begin{aligned}
& \partial \Gamma_{i}=c_{i} \gamma_{i} s_{i} \delta_{i}, \quad \gamma_{i}=\partial \Gamma_{i} \cap \sigma_{n}, \quad \delta_{i}=\partial \Gamma_{i} \cap \tau, \\
& c_{i}=\partial \Gamma_{i} \cap D_{i}, \quad s_{i}=\partial \Gamma_{i} \cap D_{i}^{\prime}, \quad\left|c_{i}\right|=\left|s_{i}\right|=2 .
\end{aligned}
$$

Пусть $\Gamma_{i}^{\prime}=D_{i} \cup \Gamma_{i} \cup D_{i}^{\prime}, \Gamma_{i}^{\prime}$ вдоль $\gamma_{i}$ не должна содержать деновских областей и полос, в противном случае в $K_{\sigma_{n}}$ будет содержаться полоса. Поэтому для каждой граничной в $\Gamma_{i}^{\prime}$ области $D \subset \Gamma_{i}$ справедливы неравенства $3 \leqslant i(D) \leqslant 5$ в $\Gamma_{3}^{\prime}$ и $d(D)=6$, в противном случае в $K_{\sigma_{n}}$ будет содержаться полоса. Удалим в $M_{n}$ все области $D_{i}$, граничашие с $\sigma_{n}$, получим кольцевую диаграмму $M_{n}^{\prime}$ с граничными циклами $\sigma_{n}^{\prime}, \tau$, являющуюся простой. Проводя для $M_{n}^{\prime}$ рассуждения, аналогичные изложенным выше, получим, что все граничные области $D$ в $M_{n}^{\prime}$ имеют $d(D)=6$ и так далее.

Теорема 4 ([1]). Пусть $G$ - группа Кокстера большого типа с множеством образующих $A,|A|<\infty$, определяемая матричей Кокстера $M_{A}$. Тогда подгруппа, порожсенная множеством $A_{J} \subset A$, есть группа Кокстера большого типа, определяемая матричей Кокстера $M_{J}$, полученной из $M_{A}$ удалением строк и столбчов, именованных образуючими из $A \backslash A J$.

Лемма 25. Пусть $M$ - кольчевая связная приведенная минимальная $R$-диаграмма вида $C(6)$ с граничными чиклами $\sigma, \tau$ группы $G$ Кокстера большого типа. Пусть $\varphi(\sigma), \varphi(\tau)$ обладают свойством $s, \varphi(\sigma)$ - слово на образующих $A_{j}, A_{j} \subset A, A-$ множество образуюших $G, \varphi(\sigma)$ не является образуюшим из $A_{j}$. Тогда $\varphi(\tau) \in G_{j}$ и слова $\varphi(\sigma)$ и $\varphi(\tau)$ сопряжены в $G_{j}$.

Доказательство. Из условий леммы следует, что кольцевая $R$-диаграмма $M$ является специальной. Если $M-n$-слойная диаграмма, $n>1$, то нетрудно убедиться в том, что метка каждой области $D \subset M$ принадлежит $G_{j}$. Пусть теперь $M$ является $(C-n)$ слойной диаграммой. Рассмотрим кольцевую диаграмму $\tilde{M}$ с граничными циклами $\sigma, \sigma_{n}$, полученную из $M$ удалением простой кольцевой поддиаграммы $M_{n} ; M_{n}$ имеет граничные циклы $\sigma_{n}, \tau$. Слово $\varphi\left(\sigma_{n}\right)$ обладает свойством $s$, поэтому $\varphi\left(\sigma_{n}\right) \in G_{j}$ и метка любого подпути, содержащегося в $\tau \cap \sigma_{n}$, является словом в $G_{j}$.

Пусть $M_{n}^{1}, M_{n}^{2}, \ldots, M_{n}^{p}$ - односвязные поддиаграммы $M_{n}$. Рассмотрим поддиаграмму $M_{n}^{j}, 1 \leqslant j \leqslant p$, которая простым путем $\chi_{j}$ соединена с предыдущей поддиаграммой, $\chi_{j}^{\prime}$ - с последующей, $\chi_{j}, \chi_{j}^{\prime} \subset \tau \cap \sigma_{n}, v_{j}=\chi_{j} \cap M_{n}^{j}, v_{j}^{\prime}=\chi_{j}^{\prime} \cap M_{n}^{j}, v_{j}, v_{j}^{\prime}$ - вершины $M_{n}^{j}$. Пусть $D_{0}, D_{0}^{\prime}$ - области из $M_{n}^{j}$ такие, что $v_{j} \in \partial D_{0}, v_{j}^{\prime} \in \partial D_{0}^{\prime}, \partial D_{0}=p_{1} p_{2} p_{3}$, где

$$
\begin{aligned}
& \partial D_{0} \cap \sigma_{n}=p_{1}, \\
& \partial D_{0} \cap \tau=p_{2}, \quad 1 \leqslant\left|p_{3}\right| \leqslant 2, \\
& p_{1} \cap p_{2}=v_{s}, \quad \partial D^{\prime}=p_{3}^{\prime} p_{2}^{\prime} p_{1}^{\prime}, \quad p_{2}^{\prime}=\partial D_{0}^{\prime} \cap \tau, \quad \partial D_{0}^{\prime} \cap \sigma_{n}=p_{1}^{\prime}, \quad 1 \leqslant\left|p_{3}^{\prime}\right| \leqslant 2 .
\end{aligned}
$$

Если $\left|p_{3}\right|=1\left(\left|p_{3}^{\prime}\right|=1\right)$, то $\left|p_{1}\right|>1\left(\left|p_{1}^{\prime}\right|>1\right)$, так как если $\left|p_{1}\right|=1\left(\left|p_{1}^{\prime}\right|=1\right)$, то получаем $R$-сократимость $\varphi(\tau)$. Отсюда следует, что $\varphi\left(D_{0}\right), \varphi\left(D_{0}^{\prime}\right) \in G_{j}$. Если $\left|p_{3}\right|=2$, $\left|\left(p_{2}\right)\right| \geqslant 3,\left|p_{1}\right|=1$, то в $K_{\tau}$ содержится полоса, так как в этом случае $D_{0}$ ограничивает слева поддиаграмму $\Gamma$, при $\left|p_{1}\right|>1 \varphi\left(D_{0}\right) \in G_{j}$. Пусть $\left|p_{3}\right|=1$ и $M_{n}^{j} \subset K_{\tau}$, в этом 
случае метка любой области $D \subset M_{n}^{j}$, в чем нетрудно убедиться, принадлежит $G_{j}$. Пусть $M_{n}^{j} \subset K_{\tau}$. Рассмотрим последовательность областей $D_{0}, D_{1}, \ldots, D_{k}$ из $M_{n}^{j}$, любые две $D_{i}, D_{i+1}, 0 \leqslant i<k$, из которых пересекаются по ребру и $i\left(D_{s}\right)=2,1 \leqslant s<k$, в $M_{n}^{j}$, но $i\left(D_{k}\right)>2$, а именно, $i\left(D_{k}\right)=3$. Очевидно, что метка каждой области $D \subset M_{n}^{j}$ принадлежит $G_{j}$.

Рассмотрим область $D_{k}$. Пусть $\partial D_{k}=p_{1} p_{2} p_{3} p_{4}$, где

$$
p_{1}=\partial D_{k} \cap \sigma_{n}, \quad p_{2}=D_{k-1} \cap D_{k}, \quad p_{3}=\partial D_{k} \cap \tau, \quad\left|p_{4}\right|=2,
$$

причем область $D_{k}$ в рассматриваемом случае ограничивает слева подлиаграмму $\Gamma$, $\partial \Gamma=\gamma \operatorname{cs} \delta$, где

$$
\partial \Gamma \cap \sigma_{n}=\gamma, \quad \partial \Gamma \cap \tau=\delta, \quad \partial \Gamma \cap D_{k}=c=p_{4}, \quad \partial \Gamma \cap D_{k}^{\prime}=s,
$$

$D_{k}^{\prime}$ - область, ограничивающая $\Gamma$ справа, $\partial D_{k}^{\prime} \cap \partial M_{n}^{j}-$ несвязное множество. Если $\left|p_{1}\right|=0$, то $i\left(D_{k}\right)=3$ в $M$ и $M$ вдоль границы $\tau$ содержит полосу. Поэтому $\left|p_{1}\right| \neq 0$ и $\varphi\left(D_{k}\right) \in G_{j}$.

Введем обозначение $\Gamma^{\prime}=D_{k} \cup \Gamma \cup D_{k}^{\prime}$. Каждая область $D \subset \Gamma^{\prime}, D \subset \Gamma$, граничащая с $\gamma$, имеет внутреннюю степень $i(D), 3 \leqslant i(D) \leqslant 5$, причем область $D$ с $i(D)=5$ граничит с простыми граничными областями $D^{\prime}, D^{\prime \prime}$, имеющими внутреннюю степень 3 либо 4, метка которых принадлежит $G_{j}$, поэтому $\varphi(D) \subset G_{j}$. Остается показать, что метка $D_{k}^{\prime}$ тоже принадлежит $G_{j}$. Пусть $\partial D_{k}=s p_{1}^{\prime} s^{\prime} p_{2}^{\prime}$, где $\partial D_{k}^{\prime} \cap \sigma_{n}=p_{2}^{\prime}, \partial D_{k}^{\prime} \cap \tau=p_{1}$. Если $\left|p_{2}^{\prime}\right| \neq 0$, то $\varphi\left(D_{k}^{\prime}\right) \in G_{j}$. Пусть $\left|p_{2}^{\prime}\right|=0$ и $s^{\prime}=2$. Тогда $D_{k}^{\prime}$ ограничивает слева следующую $\Gamma$-поддиаграмму, в этом случае $M$ содержит полосу вдоль $\tau$ (см. лемму 22 в [6]). Случай, когда $\left|p_{2}^{\prime}\right|=0,\left|s^{\prime}\right|=1$, невозможен по тем же соображениям (см. лемму 22 в [6]).

Таким образом, все области из $M_{n}$, граничащие с $\sigma_{n}$, имеют метки, принадлежащие $G_{j}$. Следовательно, метка $\sigma_{n}^{\prime}$ простой кольцевой $R$-диаграммы $M_{n}^{\prime}$, полученной из $M_{n}$ удалением областей, граничаших с $\sigma_{n}$, будет принадлежать $G_{j}$. Проводя для $M_{n}^{\prime}$ рассуждения, аналогичные вышеизложенным, убеждаемся, что все области из $M_{n}^{\prime}$, граничащие с $\sigma_{n}$, имеют метки из $G_{j}$, и так далее.

В случае, если диаграмма $M$ есть диаграмма $1^{\circ}, 2^{\circ}$, либо $3^{\circ}$, рассуждая указанным выше образом, убеждаемся в справедливости леммы.

Следствие 1. Пусть $u, v \in G, G$ - группа Кокстера большого типа, $A$ - множество образуюших $G, u, v$ обладают свойством $s$ и существует $z \in G$ такое, что $z^{-1} u z=v$. Тогда, если слово и есть слово на образующих $A_{j}, A_{j} \subset A$, и не является образующим из $A_{j}$, то $v \in G_{j}$ и существует $z^{\prime} \in G_{j}, z^{\prime}=z$ в $G$ такое, что $z^{\prime-1} u z^{\prime}=v$, где $z^{\prime-1}=\operatorname{rev}\left(z^{\prime}\right)$.

Следствие 2. Пусть $G$ - группа Кокстера большого типа на образующих $A, G_{j}-$ подгруппа $G$ на множестве образующих $A_{j} \subset A$, слово $и$ обладает свойством $s, u \in G_{j} u$ не является образующим из $A_{j}, A_{j} \subset A$, тогда $N_{G}(u)=N_{G_{j}}(u)$.

Рассмотрим кольцевые связные приведенные $R$-диаграммы группы Кокстера большого типа, содержащие $(s-i)$-области.

Лемма 26. Пусть $M$ - кольчевая связная приведенная минимальная $R$-диаграмма группь $G$ Кокстера большого типа с граничными циклами $\sigma, \tau ; \varphi(\sigma), \varphi(\tau)$ удовлетворяют условию $s$. Тогда если $M$ содержит $(s-i)$-область, то все области $M$ являются $(s-i)$ областями. 
Доказательство. Рассмотрим случай, когда кольцевая $R$-диаграмма $M$, удовлетворяющая условию леммы, содержит две $(s-i)$-области $\xi_{1}$ и $\xi_{2}$, между которыми находятся не $(s-i)$-области. Кольцевую поддиаграмму кольцевой $R$-диаграммы $M$, расположенную между областями $\xi_{1}, \xi_{2}$ обозначим через $J$. Пусть кольцевая связная $R$-диаграмма $J$ такова, что для каждой ее граничной области $D$ множество $\partial D \cap \partial J$ связно. Тогда для кольцевой $J$-диаграммы имеет место соотношение $\sum_{J}^{*}(4-i(D) \geqslant 0$, из которого следует, что существуют граничные области $D, D \in J$, с внутренней степенью $i(D) \leqslant 4$. Но каждая граничная область $D \in J$ может пересекаться с $\xi_{1}$ либо $\xi_{2}$ по ребру. Получаем противоречие.

Допустим, что $J$-кольцевая связная приведенная $R$-диаграмма имеет вид $1^{\circ}$. Тогда каждая область $D \in J$ является граничной и имеет в $J$ внутреннюю степень $i(D)=2$. Так как $d(D) \geqslant 6$, получим, что либо $\left|\varphi\left(\partial D \cap \partial \xi_{1}\right)\right|>1$, либо $\left|\varphi\left(\partial D \cap \partial \xi_{2}\right)\right|>1$. Но и то и другое противоречит условию минимальности $R$-диаграммы $M$.

Рассмотрим теперь случай, когда кольцевая связная приведенная $R$-диаграмма $J$ имеет вид $2^{\circ}$. Докажем, что $J$ содержит граничную область $D$, пересекающуюся с $\xi_{1}$ либо с $\xi_{2}$ по граничному пути, состоящему более чем из одного ребра. Пусть $\sigma^{\prime}, \tau^{\prime}-$ граничные циклы кольцевой $R$-диаграммы $J$. Допустим, что $J$ состоит из одной связной односвязной компоненты. Пусть $\gamma=\sigma^{\prime} \cap \tau^{\prime}$ и $v^{\prime}$ - начальная вершина $\gamma, v^{\prime \prime}$ - конечная вершина. Пусть $D^{\prime}$ и $D^{\prime \prime}$ - граничные области из $J$, содержащие соответственно вершины $v^{\prime}, v^{\prime \prime}$, и пусть $\partial D^{\prime} \cap \sigma^{\prime} \neq v^{\prime}, \partial D^{\prime \prime} \cap \sigma^{\prime} \neq v^{\prime \prime}$.

Если $i\left(D^{\prime}\right) \leqslant 1\left(i\left(D^{\prime \prime}\right) \leqslant 1\right)$ в $J$, то $D^{\prime}\left(D^{\prime \prime}\right)$ пересекается с $\xi_{1}$ либо с $\xi_{2}$ по пути, состоящему более чем из одного ребра, поэтому $i\left(D^{\prime}\right) \geqslant 2$ и $i\left(D^{\prime \prime}\right) \geqslant 2$ в $J$. Однако в этом случае (см. леммы 17-22 в [6]) $R$-диаграмма $J$ вдоль границы $\sigma^{\prime \prime}=\partial J \cap \sigma^{\prime}$ либо $\tau^{\prime \prime}=\partial J \cap \tau^{\prime}$ содержит либо деновскую область, либо полосу, либо $J$ содержит простую граничную область $D$, пересекающуюся с $\xi_{1}$ либо с $\xi_{2}$ по пути, состоящему более чем из одного ребра. Если $J$ состоит более чем из одной связной односвязной компоненты, то рассуждения аналогичны. Если $J$ - кольцевая $R$-диаграмма вида $3^{\circ}$, то как следует из лемм 17-22 из [6], $J$ содержит простую граничную область $D$ с $i(D) \leqslant 3$ в $J$, пересекающуюся с $\xi_{1}$ либо с $\xi_{2}$ по пути, состоящему более чем из одного ребра. Рассмотрим теперь случай, когда между граничным циклом $\sigma$ и $(s-i)$-областью $\xi$ кольцевой $R$-диаграммы $M$ содержатся области, отличные от $(s-i)$-областей, которые образуют кольцевую связную приведенную поддиаграмму $J R$-диаграммы $M$.

Пусть $\tau^{\prime}-$ внутренний граничный цикл кольцевой диаграммы $J$. Допустим, что $J-$ $n$-слойная либо $(C-n)$-слойная кольцевая $R$-диаграмма, $n>1$. Тогда каждая граничная область $D \in J$, для которой $\partial D \cap \tau^{\prime} \neq \varnothing$, пересекается с $\xi$ максимум по ребру. Поэтому внутренняя степень $i(D) \geqslant 5$ в $J$. Число областей из $J$, граничащих с $\xi$, не менее двух и $\sum_{J}^{*}(4-i(D)) \geqslant 0$, поэтому $\sum_{J}^{*}(4-i(D)) \geqslant 2$. Отсюда следует, что $R$-диаграмма содержит полосу, либо деновскую область, примыкающую к $\sigma$, что невозможно. Допустим, что $J$ - кольцевая $R$-диаграмма вида $1^{\circ}$. Так как каждая область $D \in J$ имеет в $J$ внутреннюю степень, равную 2 , и $\partial J \cap \partial D-$ несвязное множество, $D$ пересекается с $\xi$ максимум по ребру, поэтому $i(D) \leqslant 3$ в $M$, что противоречит тому, что $\sum_{\sigma}^{*}(4-i(D))=0$ в $M$. Пусть $J$ - кольцевая связная приведенная $R$-диаграмма вида $2^{\circ}$ и $J$ содержит одну связную односвязную компоненту. Пусть также $\gamma=\sigma \cap \tau^{\prime}, v^{\prime}, v^{\prime \prime}$ - соответственно начальная и конечная точки $\gamma$ и $D^{\prime}, D^{\prime \prime}-$ области из $J$, содержащие соответственно $v^{\prime}$, $v^{\prime \prime}$ и такие, что $\partial D^{\prime} \cap \sigma \neq v^{\prime}, \partial D^{\prime \prime} \cap \sigma \neq v^{\prime \prime}$. Если $i\left(D^{\prime}\right) \leqslant 1\left(i\left(D^{\prime \prime}\right) \leqslant 1\right)$ в $J$, то либо $D^{\prime}$ - деновская область в $M$, либо $D^{\prime}$ пересекается с $\xi$ по пути, содержащему более одного ребра. Поэтому $i\left(D^{\prime}\right) \geqslant 2\left(i\left(D^{\prime \prime}\right) \geqslant 2\right)$. Но в этом случае, как было отмечено выше в аналогичном случае, $R$-диаграмма $J$ вдоль границы $\sigma^{\prime \prime}=\partial J \cap \sigma^{\prime}\left(\tau^{\prime \prime}=\partial J \cap \tau^{\prime}\right)$ 
содержит либо деновскую область, либо полосу, либо простые граничные области $D_{1}, D_{2}$ с $i\left(D_{1}\right)=i\left(D_{2}\right)=3$ в $J$ и $\partial D_{1} \cap \sigma^{\prime \prime}=\partial D_{2} \cap \partial J \neq \varnothing, \partial D_{2} \cap \tau^{\prime \prime}=\partial D_{2} \cap \partial J \neq \varnothing$. Каждый из этих случаев невозможен, так как либо противоречит условию $s$, которым обладает слово $\varphi(\sigma)$, либо в $J$ содержится граничная область, которая пересекается с $\xi$ по пути, содержащему более одного ребра. Если $J$ состоит более чем из одной компоненты, то рассуждения аналогичны.

Пусть $J$ - кольцевая связная приведенная $R$-диаграмма вида $3^{\circ}$. В этом случае, как доказано в леммах 17-22 в [6], в $J$ можно выделить связную односвязную поддиаграмму $\Gamma$ с граничным циклом $\partial \Gamma=\gamma c \delta c^{\prime}, \partial \Gamma \cap \sigma=\gamma, \partial \Gamma \cap \tau^{\prime}=\delta,|c|=\left|c^{\prime}\right|=2$, заключенную в $J$ между областями $\widetilde{D}, \widetilde{D}^{\prime}$, то есть $\partial \widetilde{D} \cap \partial \Gamma=c, \partial \widetilde{D}^{\prime} \cap \partial \Gamma=c^{\prime}$. Применяя леммы 17-22 из [6], мы получаем, что $Г$ вдоль границы $\gamma$ либо $\delta$ содержит либо деновскую область, либо полосу, либо в $\Gamma$ содержатся простые граничные области $D_{1}, D_{2}$ с $i\left(D_{1}\right)=i\left(D_{2}\right)=3$ в $J$ и $\partial D_{1} \cap \sigma \neq \varnothing, \partial D_{2} \cap \tau^{\prime \prime} \neq \varnothing$. Каждый из этих случаев невозможен, так как либо противоречит условию $s$, которым обладает слово $\xi$, либо в $J$ содержится граничная область $D$, пересекающаяся с $\xi$ по пути, состоящему более чем из одного ребра.

Следствие 3. Пусть $M$ - связная приведенная минимальная $R$-диаграмма группы Кокстера большого типа с граничными чиклами $\sigma, \tau ; \varphi(\sigma), \varphi(\tau)$ удовлетворяют условию s. Тогда если $M$ состоит из $n, n>1,(s-i)$-областей, то $\varphi(\sigma)=x, \varphi(\tau)=y$, где $x, y \in\left\{a_{1}, \ldots, a_{n}\right\},\left\{a_{i}\right\}-$ множество образующих группы $G$.

Лемма 27. Пусть $M$ - кольчевая связная приведенная минимальная R-диаграмма группь Кокстера большого типа с граничными чиклами $\sigma, \tau ; \varphi(\sigma), \varphi(\tau)$ удовлетворяют условию s. Тогда если $\varphi(\sigma)=x$, где $x \in\left\{a_{1}, \ldots, a_{n}\right\},\left\{a_{i}\right\}$-множество образующих группь $G$, $\operatorname{mo} \varphi(\tau)=y, y \in\left\{a_{1}, \ldots, a_{n}\right\}$.

Доказательство. Если $M$ содержит $(s-i)$-область, то из предыдушей леммы следует, что она состоит из одних $(s-i)$-областей. Если $M$ состоит из одной $(s-i)$-области, $\varphi(\sigma), \varphi(\tau) \in G_{i j}$ то $\varphi(\sigma)=x$ и $\varphi(\tau)=y$, где $x, y \in\left\{a_{1}, \ldots, a_{n}\right\},\left\{a_{i}\right\}-$ множество образующих группы $G$. Допустим, что $M$ не содержит $(s-i)$-области, тогда $M$ является $R$-диаграммой с условием $C(6)$. Если $M-(C-n)$-слойная $R$-диаграмма с $n>1$, то $M$ содержит простую граничную область $D$ такую, что $\partial D \cap \sigma \neq \varnothing$ и $i(D)=3$. Но тогда $|\varphi(D)| \leqslant 4$, что невозможно. Если $M-(C-1)$-слойная $R$-диаграмма, то $M$ содержит простую граничную область $D$ такую, что $\partial D \cap \sigma \neq \varnothing$ и $i(D)=3$. Получили рассмотренный выше случай.

Допустим, что $M-n$-слойная $R$-диаграмма с $n>1$. Легко заметить, что метки всех областей из $K_{\sigma}$-слоя принадлежат одной группе $G_{i j}$. Поэтому $\varphi\left(\sigma_{1}\right)=y$, где $x, y \in\left\{a_{i}, a_{j}\right\}$ и $K_{\sigma}$-слой является $(s-i)$-областью.

Случай, когда $M-1$-слойная $R$-диаграмма, аналогичен предыдущему. Пусть $M-$ $R$-диаграмма типа $2^{\circ}$, каждая область $D$ которой является граничной, и пересечение $\partial D \cap \partial M-$ несвязное множество. Тогда $M$ состоит из конечного числа связных односвязных диаграмм. Рассмотрим для простоты случай, когда $M$ состоит из одной односвязной $R$-диаграммы $M_{1}$. Тогда $\partial M_{1} \cap \sigma=\sigma_{1}, \partial M_{1} \cap \tau=\tau_{1}$ и метки $\varphi\left(\sigma_{1}\right), \varphi\left(\tau_{1}\right)$ принадлежат некоторой группе $G_{i j}$. Области $D_{1}, D_{2}, \ldots, D_{n}$, составляющие $M_{1}$, образуют полосу, что невозможно, так как $\varphi(\tau)$ обладает свойством $s$. Если в $R$-диаграмме $M$ типа $2^{\circ}$ содержится простая граничная область, то в $M$ можно выделить односвязную поддиаграмму $\Gamma, \partial \Gamma=\gamma c_{1} \delta c_{2}$, где $\gamma=\partial \Gamma \cap \sigma, \delta=\partial \Gamma \cap \tau,\left|c_{1}\right|=\left|c_{2}\right|=2$, в которой содержится простая граничная область $D$ с $i(D)=3$ в $M$ и $\partial D \cap \sigma \neq \varnothing$, что невозможно. Если $M$ является $R$-диаграммой типа $3^{\circ}$, то, как и в предыдущем случае, можно выделить 
односвязную поддиаграмму $\Gamma$, содержащую простую граничную область $D$ с $i(D)=3$ в $M$ и $\partial D \cap \sigma \neq \varnothing$. И наконец, диаграмма типа $1^{\circ}$ есть 1 -слойная $R$-диаграмма.

Лемма 28. Пусть $G$ - группа Кокстера большого типа с множеством образующих $A$. Если $v_{0} \in G, v_{0} \neq 1$ в $G$ и $v_{0}-R$-приведенное спечиально $R$-приведенное слово, равное слову из подгруппы $G_{j}$ с образующими $A_{j}, A_{j} \subset A$, то $v_{0}-$ слово на образующих $A_{j}$.

Доказательство. Пусть слово $v_{0}$, удовлетворяющее условиям леммы, содержит буквы из множества $B_{j}=A \backslash A_{j}$ и равно некоторому слову $u_{0}^{-1} \in G_{j}$. Пусть $v_{0}=v_{01} v v_{02}$, где $v_{01}, v_{02} \in G_{j}, v$ начинается и оканчивается на буквы из $B_{j}$. Преобразуем равенство $v_{0}=u_{0}^{-1}$ в равенство $v=u^{-1}$, где $u^{-1}=v_{01}^{-1} v_{0}^{-1} v_{02}^{-1}, u^{-1} R$-приведено и специально $R$-приведено. Слово $u v$ является приведенным и циклически приведенным, и $u v=1$ в $G$. Тогда существует связная односвязная приведенная минимальная $R$-диаграмма $M$ с граничным циклом $\alpha \beta$, где $\varphi(\alpha)=u, \varphi(\beta)=v$. Допустим, что $M$ состоит из одной области $D$. Тогда $\alpha$ является ребром $D$, поэтому $|u|=1$ и слово $v$ является $R$-сократимым. Допустим, что $M$ не содержит $n$ областей $n>1$. Тогда для $M$ выполняется формула кривизны $\sum_{M}^{*}(4-i(D)) \geqslant 6$. Тогда $\alpha \cap \beta=\left\{O_{1}, O_{2}\right\}$, где $O_{1}=H(\alpha)=K(\beta)$, $\mathrm{O}_{2}=K(\alpha)=H(\beta)$. Покажем, что $M$ не содержит деновских областей. Допустим противное. Пусть $D$ - деновская область $M$. Тогда $\partial D$ содержит либо точку $O_{2}$, либо $O_{1}$. Допустим, что $O_{2} \in \partial D$, тогда $\partial D \cap \partial M=\gamma \delta$, где $\partial D \cap \alpha=\delta, \partial D \cap \beta=\gamma$. Учитывая строение метки $\varphi(\partial D)$ и условие $u \in G_{j}$, получаем, что $|\varphi(\delta)|=1$ и $|\varphi(\gamma)| \geqslant 3$. Так как $v$ является $R$-несократимым, справедливы равенства $i(D)=2$ и $|\varphi(\gamma)|=3$. В этом случае в $M$ можно выделить связную односвязную поддиаграмму $\Gamma$, ограниченную в $M$ слева областью $D$, справа областью $D^{\prime}, \partial \Gamma=c \alpha_{1} c^{\prime} \beta_{1}$, где $\partial \Gamma \cap \alpha=\alpha_{1}, \partial \Gamma \cap \beta=\beta_{1}$, $\partial D \cap \Gamma=c, \partial D^{\prime} \cap \Gamma=c^{\prime},|c|=\left|c^{\prime}\right|=2$. Обозначим $M_{1}$ объединение $D, \Gamma$ и $D^{\prime}$ и через $D_{1}, D_{2}, \ldots, D_{p}$ - области, которые содержатся в $\Gamma$ и граничат с $\beta_{1}$. Как показано в леммах 19-22 в [6], все они являются простыми в $M_{1}$ и $3 \leqslant i\left(D_{j}\right) \leqslant 5$ для всех $j, 1 \leqslant j \leqslant p$, кроме того, существует $m, 1 \leqslant m \leqslant p$, такое, что $i\left(D_{1}\right)=i\left(D_{2}\right)=\ldots=i\left(D_{m-1}\right)=4$, $i\left(D_{m}\right)=3$ в $M_{1}$, а также и в $M$. Поэтому в $M$ области $D, D_{1}, \ldots, D_{m}$ образуют полосу, то есть $v$ специально $R$-сократимо, что невозможно. Тогда $M$ содержит как минимум три непересекающиеся полосы, что невозможно.

Пусть $M$ - кольцевая $R$-диаграмма, $v$ - произвольная точка, принадлежащая некоторому замкнутому ребру $e \in M, e=e^{\prime} e^{\prime \prime}, e^{\prime} \cap e^{\prime \prime}=v$. Тогда замкнутый путь $l \in M \mathrm{c}$ начальной и конечной точкой $v$

$$
l=e^{\prime-1} e_{1} \ldots e_{n} t
$$

где $t=e^{\prime}$, либо $t=e^{\prime \prime-1}$, либо $l=e^{\prime \prime} e_{1}^{\prime} \ldots e_{n}^{\prime} t^{\prime}$, где $t^{\prime}=e^{\prime}$, либо $t^{\prime}=e^{\prime \prime-1}$ назовем циклическим в $M$, если $l$ гомотопен $\tau$, соответственно $\sigma$. Кратчайший из всех циклических путей кольцевой $R$-диаграммы $M$, проходящих через некоторую точку $v$, принадлежащую ребру $e, e \in M$, назовем циклическим геодезическим путем с началом и концом B $v$.

Теорема 5 ([2]). В группе Кокстера большого типа разрешима проблема сопряженности слов.

Теорема 6. Централизатор конечно порожденной подгруппы $H$ группы Кокстера большого типа $G$ есть конечно порожденная подгруппа и существует алгоритм, выписывающий образующие централизатора. 
Доказательство. Пусть $M$ - кольцевая $R$-диаграмма, $v$ - произвольная точка, принадлежащая некоторому замкнутому ребру $e \in M, e=e^{\prime} e^{\prime \prime}, e^{\prime} \cap e^{\prime \prime}=v$. Тогда замкнутый путь $l \in M$ с начальной и конечной точкой $v$

$$
l=e^{\prime-1} e_{1} \ldots e_{n} t
$$

где $t=e^{\prime}$, либо $t=e^{\prime \prime-1}$, либо $l=e^{\prime \prime} e_{1}^{\prime} \ldots e_{n}^{\prime} t^{\prime}$, где $t^{\prime}=e^{\prime}$ либо $t^{\prime}=e^{\prime \prime-1}$, назовем циклическим в $M$, если $l$ гомотопен $\tau$, соответственно $\sigma$. Кратчайший из всех циклических путей кольцевой $R$-диаграммы $M$, проходящих через некоторую точку $v$, принадлежащую ребру $e, e \in M$, назовем циклическим геодезическим путем с началом и концом B $v$.

Пусть $u, v$ - слова, принадлежащие группе Кокстера большого типа, заданной на множестве образующих $A=\left\{a_{1}, a_{2}, \ldots, a_{n}\right\}$ с помощью матрицы Кокстера $\left(m_{i j}\right)_{i, j \in J}$, $|J|=n$. Допустим, что слова $u, v$ являются тупиковыми и сопряжены в $G$. Тогда существует кольцевая связная приведенная $R$-диаграмма с граничными циклами $\sigma, \tau$, метками которых являются соответственно слова $u, v$.

Пусть $u=x, x \in\left\{a_{i}\right\}_{i=1, \ldots, n}$, тогда из предыдущей леммы следует, что $v=y$, $y \in\left\{a_{i}\right\}, i=1, \ldots, n$, и диаграмма сопряженности этих слов состоит из $(s-i)$-областей. Пусть $\sigma_{0}=\sigma, \sigma_{1}, \ldots, \sigma_{k}=\tau$ - граничные циклы $R$-диаграмм, полученных из $M=M_{0}$ последовательным удалением $(s-i)$-областей. Но тогда $\varphi\left(\sigma_{i}\right)=x_{i}, x_{i} \in\left\{a_{i}\right\}_{i=1, \ldots, n}$ и любые два элемента $x_{i-1}, x_{i}, i=1, \ldots, n$, где $x=x_{0}, x_{n}=y$ сопряжены в $G_{x_{i-1} x_{i}}$ максимальным куском определяющего соотношения группы $G_{x_{i-1}} x_{i}$. Пусть

$$
m_{0}=\max \left\{m_{i j}<\infty \mid m_{i j} \text { элементы матрицы Кокстера }\right\} \text {. }
$$

Тогда длина $d$ произвольного циклического геодезического пути из $M$ заключена в пределах $|u| \leqslant d \leqslant|u|+2 m_{0}$.

Пусть слова $u, v$ не являются образующими $G$. В этом случае $u, v$ будут метками граничных циклов кольцевой $R$-диаграммы с условием $C(6)$. Слово $u$ сопряжено с $v$, и $u, v$ обладают свойством $s$ и являются тупиковыми, поэтому $|u|=|v|$.

Если $M-n$-слойная диаграмма, $n>1$, то длина циклического геодезического пути $d$ не превосходит $|u|$. Пусть $M-(C-n)$-слойная $R$-диаграмма, $n>1$; выделим в ней максимальную $n$-слойную диаграмму $M^{\prime}$ и, удалив ее из $M$, получим диаграмму $M_{n}$. В $M^{\prime}$ длина $d$ произвольного циклического пути не превосходит $|u|$. Укажем границы изменения длины циклического геодезического пути для $M_{n}$-диаграммы. Любая область $D \in M_{n}$ имеет $d(D)=6$, тогда максимальная длина метки областей $D \in M_{n}$ не превзойдет $L=6$. Используя формулу Р. Линдона (см. [3]) о числе площадей в односвязной $R$-диаграмме, получим что длина $d$ циклического геодезического пути заключена в пределах

$$
|u| \leqslant d \leqslant q(2|u| L+4 L)^{2}(3 L+2|u|)+2 L .
$$

Пусть $w_{1}, w_{2}, \ldots, w_{m}$ - образующие $H, H<G$; считаем, что $w_{1}=w_{10}$ - тупиковое слово, удовлетворяющее условию $s$ и $w_{i}=c_{i} w_{i 0} c_{i}^{-1}$ для всех $i, 1<i \leqslant m$, где $w_{i 0}$ тоже обладает свойством $s$ и является тупиковым; $\Delta\left(w_{i 0}, w_{i 0}\right)$ - кольцевая связная приведенная $R$-диаграмма сопряженности слова $w_{i 0}$ слову $w_{i 0}$. Введем обозначения $c=\max \left\{\left|c_{1}\right|, \ldots,\left|c_{m}\right|\right\}$, где $\left|c_{1}\right|=0, L=2\left(m_{0}+1\right)$ и $S\left(w_{i}, w_{i}\right), i=1, \ldots, m,-$ множество слов, длины $d_{i}$ которых заключены в пределах

$$
\left|w_{i 0}\right| \leqslant d_{i} \leqslant q\left(2\left|w_{i}\right|+2 L\right)^{2}\left(2\left|w_{i}\right|+3 L\right)+2 L+2|c| .
$$


Рассмотрим последовательность

$$
w_{1}^{(0)}, \ldots, w_{n}^{(0)}, H_{1}, w_{1}^{(1)}, \ldots, w_{n}^{(1)}, H_{2}, \ldots, H_{p}, w_{1}^{(p)}, \ldots, w_{n}^{(p)}, \ldots
$$

где $H_{i}^{-1} w_{1}^{(i-1)} H_{i}=w_{1}^{(i)}, \ldots, H_{i}^{-1} w_{n}^{(i-1)} H_{i}=w_{n}^{(i)}, H_{i} \in\left\{a_{i}\right\}$ для всех $i, 1 \leqslant i \leqslant p$, $w_{j}^{(s)} \in S\left(w_{j}, w_{j}\right)$ для всех $j, s, 1 \leqslant j \leqslant n, 1 \leqslant s \leqslant p$.

Последовательность (1) называется базисной. Базисную последовательность (1) назовем фундаментальной, если для всех $j, s, 0 \leqslant j<s<p$, наборы $\left(w_{1}^{(j)}, \ldots, w_{n}^{(j)}\right)$, $\left(w_{1}^{(s)}, \ldots, w_{n}^{(s)}\right)$ различны и существует целое $v, 0 \leqslant v<p$, такое что

$$
w_{1}^{(v)}=w_{1}^{(p)}, \ldots, w_{n}^{(v)}=w_{n}^{(p)} .
$$

Лемма 29. Если последовательность (1) фундаментальная, то слово

$$
H_{1} H_{2} \ldots H_{p} H_{v}^{-1} \ldots H_{1}^{-1}
$$

принадлежит централизатору подгруппы $H$.

Доказательство очевидно.

Слово $H_{1} H_{2} \ldots H_{p} H_{v}^{-1} \ldots H_{1}^{-1}$, связанное с фундаментальной последовательностью (1), назовем базисным словом.

Лемма 30. Если последовательность (1) является фундаментальной базисной последовательностью, то

$$
p \leqslant|S|=\left|S\left(w_{1}, w_{1}\right)\right| \ldots\left|S\left(w_{n}, w_{n}\right)\right| .
$$

Лемма 31. Число фундаментальных базисных последовательностей конечно.

Доказательство очевидно.

Лемма 32. Пусть $F \in C_{G}(H), C_{G}(H)$ - чентрализатор $H$ в $G$. Тогда существует разбиение $F$ в произведение образуючих

$$
F=H_{1} H_{2} \ldots H_{m}, \quad H_{i} \in\left\{a_{i}\right\}, \quad i=1, \ldots, m,
$$

и базисная последовательность, связанная с данными разбиением $F$, то есть

$$
w_{1}^{(0)}, \ldots, w_{n}^{(0)}, H_{1}, w_{1}^{(1)}, \ldots, w_{n}^{(1)}, H_{2}, \ldots, H_{m}, w_{1}^{(m)}, \ldots, w_{n}^{(m)}
$$

Доказательство. Пусть $F \in C_{G}(H), F \neq 1$, тогда имеет место система соотношений

$$
F^{-1} w_{1} F=w_{1}, \quad c_{2} F^{-1} c_{2}^{-1} w_{20} c_{2} F c_{2}^{-1}=w_{20}, \ldots, c_{n} F^{-1} c_{n}^{-1} w_{n 0} c_{n} F c_{n}^{-1}=w_{n 0} .
$$

Пусть для всех $i, 1<i \leqslant n$, существуют $X_{i}, Y_{i}, F_{i}$ такие, что $F \equiv X_{i} F_{i} Y_{i}$, где $\equiv$ означает графическое равенство, $c_{i}=c_{i}^{\prime} X_{i}^{-1}=c_{i}^{\prime \prime} Y_{i}$. В результате получаем равенства

$$
F^{-1} w_{1} F=w_{1}, \quad c_{i}^{\prime \prime} F_{i}^{-1} c_{i}^{\prime-1} w_{i 0} c_{i}^{\prime} F_{i} c_{i}^{\prime \prime-1}=w_{i 0}, \quad 1<i \leqslant n,
$$

где каждое из слов $c_{i}^{\prime} F_{i} c_{i}^{\prime \prime-1}$ несократимо.

Рассмотрим кольцевые приведенные $R$-приведенные, специально $R$-приведенные $R$-диаграммы $\Delta\left(w_{i 0}, w_{i 0}\right)$ с граничными циклами $\sigma^{(i 0)}, \tau^{(i 0)}$, где $\varphi\left(\sigma^{(i 0)}\right)=w_{i 0}$, 
$\varphi\left(\tau^{(i 0)}\right)=w_{i 0}^{-1}, 1 \leqslant i \leqslant n$ (при $i=1, w_{10}=w_{1}$ ), каждая из которых, соответственно, является диаграммой сопряженности для $i$-го соотношения.

Обозначим через $O^{(i 0)}$ начальную точку на $\sigma^{(i 0)}$ и через $O^{\prime(i 0)}$ - начальную точку на $\tau^{(i 0)}, i=1, \ldots, n$. Тогда в диаграмме $\Delta\left(w_{i 0}, w_{i 0}\right), i=1, \ldots, n$, содержится путь $\eta_{i}$, $\alpha\left(\eta_{i}\right)=O^{(i 0)}, \omega\left(\eta_{i}\right)={O^{\prime}}^{(i 0)}$ c $\varphi\left(\eta_{1}\right)=F, \varphi\left(\eta_{i}\right)=c_{i}^{\prime} F_{i} c_{i}^{\prime \prime-1}, i=2, \ldots, n$, где $\alpha(\eta), \omega(\eta)$ - соответственно начало и конец пути $\eta$. Пусть

$$
\varphi\left(\eta_{1}\right)=F=H_{1}^{(1)} H_{2}^{(1)} \ldots H_{m(1)}^{(1)}
$$

- разбиение $F$ в диаграмме $\Delta\left(w_{10}, w_{10}\right)$ на образующие. Тогда

$$
F \equiv X_{i} F_{i} Y_{i}=H_{1}^{(1)} \ldots H_{\alpha_{(i)}^{(1)}}^{(1)} H_{\alpha_{(i)+1}^{(1)}}^{(1)} \ldots H_{\beta_{(i)}^{(1)}}^{(1)} H_{\beta_{(i)+1}^{(1)}}^{(1)} \ldots H_{m(1)}^{(1)}
$$

где

$$
X_{i}=H_{1}^{(1)} \ldots H_{\alpha_{(i)}^{(1)}}^{(1)}, \quad F_{i}=H_{\alpha_{(i)+1}^{(1)}}^{(1)} \ldots H_{\beta_{(i)}^{(1)}}^{(1)}, \quad Y_{i}=H_{\beta_{(i)+1}^{(1)}}^{(1)} \ldots H_{m(1)}^{(1)}
$$

С другой стороны, каждое $\varphi\left(\eta_{i}\right)=c_{i}^{\prime} F_{i} c_{i}^{\prime \prime-1}$ в соответствующей диаграмме $\Delta\left(w_{i 0}, w_{i 0}\right)$ разбивается на образующие

$$
\varphi\left(\eta_{i}\right)=H_{1}^{(i)} \ldots H_{\alpha_{(i)}^{(1)}}^{(i)} H_{\alpha_{(i)+1}^{(i)}}^{(i)} \ldots H_{\beta_{(i)}^{(i)}}^{(i)} H_{\beta_{(i)+1}^{(i)}}^{(i)} \ldots H_{m(i)}^{(i)},
$$

где

$$
c_{i}^{\prime}=H_{1}^{(i)} \ldots H_{\alpha_{(i)}^{(i)}}^{(i)}, \quad F_{i}=H_{\alpha_{(i)+1}^{(i)}}^{(i)} \ldots H_{\beta_{(i)}^{(i)}}^{(i)}, \quad c_{i}^{\prime \prime-1}=H_{\beta_{(i)+1}^{(i)}}^{(i)} \ldots H_{m(i)}^{(i)} .
$$

Отсюда следуют соотношения

$$
F_{i}=H_{\alpha_{(i)+1}^{(1)}}^{(1)} \ldots H_{\beta_{(i)}^{(1)}}^{(1)}=H_{\alpha_{(i)+1}^{(i)}}^{(i)} \ldots H_{\beta_{(i)}^{(i)}}^{(i)} .
$$

Заметим, что разбиение $X_{i}$ определяется разбиением $F$. Аналогично, разбиение $Y_{j}$ также определяется разбиением $F$. Следовательно, $X_{i}, Y_{j}$ на искомое разбиение $F$ не влияют. В качестве искомого возьмем разбиение $\varphi\left(\eta_{1}\right)=F$ в диаграмме $\Delta\left(w_{10}, w_{10}\right)$. Получим разбиение $F=H_{1} H_{2} \ldots H_{m}$ и последовательность $w_{1}^{(0)}, \ldots, w_{n}^{(0)}, H_{1}, w_{1}^{(1)}, \ldots, w_{n}^{(1)}$, $H_{2}, \ldots, H_{m}, w_{1}^{(m)}, \ldots, w_{n}^{(m)}$, связанную с полученным разбиением и удовлетворяющую следующим условиям:

$$
H_{i}^{-1} w_{1}^{(i-1)} H_{i}=w_{1}^{(i)}, \ldots, H_{i}^{-1} w_{n}^{(i-1)} H_{i}=w_{n}^{(i)}, H_{i} \in\left\{a_{i}\right\}
$$

для всех $i, 1 \leqslant i \leqslant m$, и $w_{j}^{(s)} \in S\left(w_{j}, w_{j}\right)$ для всех $j, s, 1 \leqslant j \leqslant n, 1 \leqslant s \leqslant m$.

Лемма 33. Множество всех базисных слов порождает чентрализатор подгруппы $H$.

Доказательство очевидно.

Из лемм 29-33 следует справедливость теоремы 6.

Следствие 4. Централизатор произвольного элемента $w \in G$ есть конечно порожденная подгруппа в $G$ и существует алгоритм, выписывающий образующие этого чентрализатора. 
Заметим, что централизатор элемента в группах Кокстера большого типа в общем случае не является циклической подгруппой. Рассмотрим группу

$$
\begin{aligned}
& G=\langle a, b, c, d ; a b a=b a b, a c a=c a c, \\
& \left.\quad a d a=d a d, b c b=c b c, b d b=d b d, c d c=d c d, a^{2}=1, b^{2}=1, c^{2}=1, d^{2}=1\right\rangle
\end{aligned}
$$

и слово $w=d a c d b c a b$ из $G$. Слово $w$ обладает свойством $s$ и является тупиковым. Следовательно, оно не является равным или сопряженным ни одному слову с числом образующих, меньшим трех. И так как $w$ удовлетворяет равенству $a w a=w$, централизатор $w$ не является циклической подгруппой.

Заметим также, что в общем случае группы Кокстера большого типа не являются гиперболическими группами. Рассмотрим группу

$$
G=\left\langle a, b, c ; a b a=b a b, a c a=c a c, b c b=c b c, a^{2}=1, b^{2}=1, c^{2}=1\right\rangle .
$$

Данная группа содержит свободную абелеву подгруппу

$$
\left\langle(a b c)^{2},(b a c)^{2} ;(a b c)^{2}(b a c)^{2}=(a b c)^{2}(b a c)^{2}\right\rangle,
$$

а потому не может быть гиперболической. Действительно,

$$
\begin{aligned}
\text { bacbacabcabc } & =\text { bacbcacbcabc = babcbacbcabc = abacbacbcabc } \\
& =a b a c b a b c b a b c=a b a c a b a c b a b c=a b c a c b a c b a b c \\
& =a b c a c b a c a b a c=a b c a c b c a c b a c=a b c a b c b a c b a c .
\end{aligned}
$$

Теорема 7. В группе Кокстера большого типа разрешима проблема обобщенной сопряженности слов.

Доказательство. Доказательство данной теоремы сведем к доказательству теоремы 6.

Пусть даны множества слов $w_{1}, w_{2}, \ldots, w_{m}$ и $v_{1}, v_{2}, \ldots, v_{m}$. Необходимо установить, существует ли $z, z \in G$, такое, что $\&_{i=1}^{m}\left(z^{-1} w_{i} z=v_{i}\right)$.

Пусть слова $w_{1}=w_{10}, v_{1}=v_{10}$ обладают свойством $s$ и являются тупиковыми. Пусть

$$
w_{i}=a_{i} w_{i 0} a_{i}^{-1}, \quad v_{i}=b_{i} v_{i 0} h_{i}^{-1}, \quad i=2, \ldots, m
$$

где $w_{i 0}, v_{i 0}$ обладают свойством $s$ и являются тупиковыми. Если предположить, что эти множества сопряжены, то $\left|w_{i 0}\right|=\left|v_{i 0}\right|$ для всех $i=1, \ldots, m$, и если какое-то из $w_{i 0}$ есть образующий $x \in\left\{a_{i}\right\}$, то сопряженное ему слово $v_{i 0}$ - тоже образующий $y \in\left\{a_{i}\right\}$. Пусть $\Delta\left(w_{i 0}, v_{i 0}\right)$ - кольцевая связная приведенная $R$-диаграмма сопряженности слов $w_{i 0}, v_{i 0}$,

$$
|a|=\max \left\{\left|a_{1}\right|,\left|a_{2}\right|, \ldots,\left|a_{m}\right|\right\}, \quad|b|=\max \left\{\left|b_{1}\right|,\left|b_{2}\right|, \ldots,\left|b_{m}\right|\right\},
$$

где $\left|a_{1}\right|=\left|h_{1}\right|=0, L=2\left(m_{0}+1\right)$.

Обозначим через $S\left(w_{i}, v_{i}\right), i=1, \ldots, m$, множество всех слов, длина $d_{i}$ которых заключена в пределах

$$
\left|w_{i 0}\right| \leqslant d_{i} \leqslant q\left(\left(\left|w_{i}\right|+\left|v_{i}\right|\right) L+2 L\right)^{2}\left(\left(2\left|w_{i}\right|+\left|v_{i}\right|\right) L+3 L\right)+2 L+(|a|+|b|) .
$$

Для всех $i, i=1, \ldots, n$, положим $w_{i}=w_{i}^{(0)}$ и рассмотрим базисные последовательности, соответствующие множеству слов $w_{1}^{(0)}, \ldots, w_{n}^{(0)}$,

$$
w_{1}^{(0)}, \ldots, w_{n}^{(0)}, H_{1}, w_{1}^{(1)}, \ldots, w_{n}^{(1)}, H_{2}, \ldots, H_{k}, w_{1}^{(k)}, \ldots, w_{n}^{(k)},
$$


где $w_{i}^{(j)} \in S\left(w_{i}, v_{i}\right)$ для всех $i, 1 \leqslant i \leqslant n$, и $j, 0 \leqslant j \leqslant k$.

Базисная последовательность (2) называется особой, если она не содержит фундаментальную последовательность либо является пустой, то есть все $H_{i}=1$. Слово $H_{1} H_{2} \ldots H_{k}$, соответствующее особой базисной последовательности, назовем особым базисным словом.

Если $w_{1}^{(k)}=v_{1}, \ldots, w_{n}^{(k)}=v_{n}$ в базисной последовательности (2), то слова $w_{1}, \ldots, w_{n}$ обобщенно сопряжены словам $v_{1}, \ldots, v_{n}$.

Лемма 34. Если (2) является особой базисной последовательностью, то $k \leqslant|S|=$ $\left|S\left(w_{1}, v_{1}\right)\right| \ldots\left|S\left(w_{n}, v_{n}\right)\right|$.

Лемма 35. Число особых базисных последовательностей конечно.

Доказательство очевидно.

Лемма 36. Пусть $F$-какое-то решение системы $\&_{i=1}^{n}\left(z^{-1} w_{i} z=v_{i}\right)$, тогда существует разбиение $F$ в произведение кусков $H_{1} H_{2} \ldots H_{m}, H_{i} \in\left\{a_{i}\right\}, i=1, \ldots, m, u$ базисная последовательность, связанная с данным разбиением $F$

$$
w_{1}^{(0)}, \ldots, w_{n}^{(0)}, H_{1}, w_{1}^{(1)}, \ldots, w_{n}^{(1)}, H_{2}, \ldots, H_{m}, w_{1}^{(m)}, \ldots, w_{n}^{(m)},
$$

где $w_{1}^{(m)}=v_{1}, \ldots, w_{n}^{(m)}=v_{n}$.

Доказательство аналогично доказательству леммы 32.

Лемма 37. Пусть $F-$-какое-то решение системы $\alpha_{i=1}^{n}\left(z^{-1} w_{i} z=v_{i}\right) u(3)-$ базисная последовательность, соответствующая данному разбиению $F$. Тогда из последовательности (3) можно выделить особую подпоследовательность такую, что соответствуюшее ей базисное слово $F$ является решением системы.

Доказательство. Если система $\&_{i=1}^{n}\left(z^{-1} w_{i} z=v_{i}\right)$ такова, что $w_{i} \equiv v_{i}$ для всех $i=1, \ldots, n$, то в качестве особой базисной подпоследовательности рассмотрим пустую подпоследовательность с $F \equiv 1$. Если последовательность (3) не содержит фундаментальных подпоследовательностей, то $F^{\prime}=F$. Если (3) не является особой, то сушествуют целые числа $v, k, 0 \leqslant v<k<m$, такие, что $w_{1}^{(0)}, \ldots, w_{n}^{(0)}, H_{1}, \ldots, H_{v}, w_{1}^{(v)}, \ldots, w_{n}^{(v)}$, $H_{v+1}, \ldots, H_{k}, w_{1}^{(k)}, \ldots w_{n}^{(k)}$ является фундаментальной последовательностью. Вычеркнув из (3) подпоследовательность $H_{v+1}, w_{1}^{(v+1)}, \ldots, w_{n}^{(v+1)} H_{v+2}, \ldots, H_{k}, w_{1}^{(k)}, \ldots, w_{n}^{(k)}$, получим базисную последовательность слов, являющуюся решением системы. Если полученная базисная последовательность не является особой, то применим к ней указанный выше процесс.

Из лемм 34-37 следует справедливость теоремы 7.

Теорема 8. Пусть $G$ - конечно определенная группа Кокстера большого типа и $\left\{w_{i}\right\}_{i=1, \ldots,, m}, \quad\left\{v_{i}\right\}_{i=1, \ldots, m}-$ слова из G. Если $F$ - какое-то решение системы $\boldsymbol{\alpha}_{i=1}^{n}\left(z^{-1} w_{i} z=v_{i}\right)$, то множество слов $C_{G}(H) F$, где $C_{G}(H)$ - чентрализатор подгруппы $H$, порожденной словами $\left\{w_{i}\right\}_{i=1, \ldots, m}$, является множеством всех решений системы.

Теорема 9. Существует алгоритм, позволяющий для любого конечного множества слов из конечно определенной группы Кокстера $G$ большого типа выписать образующие их нормализатора.

Доказательство очевидно. 


\section{Список литературы}

1. Appel K., On Artin groups and Coxeter groups of large type. Contemp. Math. (1984) 33, 50-78.

2. Безверхний В. Н., Добрынина И. В., Решение проблемы сопряженности слов в группах Кокстера большого типа. Чебымевский сборник (2003) 4, №1(5), 10-33.

3. Линдон Р., Шупп П., Комбинаторная теория групп. Наука, Москва, 1980.

4. Безверхний В. Н., Решение проблемы сопряженности слов в группах Артина большого типа. В сб.: Алгоритмические проблемы теорий групп и полугрупп. Межвузовский сборник научных трудов. Тульский гос. пед. инст., Тула, 1986, с. 26-61.

5. Гриндлингер М. Д., О проблеме сопряженности и совпадений с антицентром в теории групп. Сиб. матем. журнал. (1996) 7, 785-803.

6. Безверхний В. Н., О нормализаторах элементов в группах. В сб.: Алгоритмические проблемы теории групп и полугрупп. Межвузовский сбориик паучиых трудов. Тульский гос. пед. инст., Тула, 1994.

7. Безверхний В. Н., Решение обобщенной проблемы сопряженности слов в $C(p) \& T(q)$-группах. Изв. Тульского гос. унив. (1998) 4, №3, 5-13.

8. Безверхний В. Н., Решение проблемы обобщенной сопряженности слов в группах Артина большого типа. Фундаментальная и прикладная матем. (1999) 5, №1, 1-38.

Статья поступила 14.10.2003. 